\title{
Dynamic PET Reconstruction Using Wavelet Regularization With Adapted Basis Functions
}

\author{
Jeroen Verhaeghe*, Dimitri Van De Ville, Member, IEEE, Ildar Khalidov, Yves D'Asseler, Senior Member, IEEE, \\ Ignace Lemahieu, Member, IEEE, and Michael Unser, Fellow, IEEE
}

\begin{abstract}
Tomographic reconstruction from positron emission tomography (PET) data is an ill-posed problem that requires regularization. An attractive approach is to impose an $\ell_{1}$-regularization constraint, which favors sparse solutions in the wavelet domain. This can be achieved quite efficiently thanks to the iterative algorithm developed by Daubechies et al., 2004. In this paper, we apply this technique and extend it for the reconstruction of dynamic (spatio-temporal) PET data. Moreover, instead of using classical wavelets in the temporal dimension, we introduce exponential-spline wavelets ( $E$-spline wavelets) that are specially tailored to model time activity curves (TACs) in PET. We show that the exponential-spline wavelets naturally arise from the compartmental description of the dynamics of the tracer distribution. We address the issue of the selection of the "optimal" $E$-spline parameters (poles and zeros) and we investigate their effect on reconstruction quality. We demonstrate the usefulness of spatio-temporal regularization and the superior performance of $E$-spline wavelets over conventional Battle-Lemarié wavelets in a series of experiments: the 1-D fitting of TACs, and the tomographic reconstruction of both simulated and clinical data. We find that the $E$-spline wavelets outperform the conventional wavelets in terms of the reconstructed signal-to-noise ratio (SNR) and the sparsity of the wavelet coefficients. Based on our simulations, we conclude that replacing the conventional wavelets with $E$-spline wavelets leads to equal reconstruction quality for a $40 \%$ reduction of detected coincidences, meaning an improved image quality for the same number of counts or equivalently a reduced exposure to the patient for the same image quality.
\end{abstract}

Index Terms-Differential system, $E$-spline wavelets, $\ell_{1}$-regularization, spatio-temporal positron emission tomography (PET) reconstruction, time-activity-curves.

Manuscript received January 31, 2008; revised March 13, 2008. This work was supported in part by the Ghent University, in part by the Research Foundation-Flanders (FWO), in part by an IAP grant from the Belgian federal government, in part by the Center for Biomedical Imaging (CIBM) of the Geneve-Lausanne Universities, in part by the EPFL, in part by the Foundations Leenaards and Louis-Jeantet, and in part by the Swiss National Science Foundation under Grant 200020-109415. Asterisk indicates corresponding author.

*J. Verhaeghe is with the Department of Electronics and Information Systems, MEDISIP, Ghent University-IBBT-IBiTech, De Pintelaan 185 block B, B-9000 Ghent, Belgium (e-mail: jeroen.verhaeghe@ugent.be).

D. Van De Ville, I. Khalidov, and M. Unser are with the Biomedical Imaging Group, Ecole Polytechnique Fédérale de Lausanne (EPFL), CH-1015 Lausanne, Switzerland.

Y. D'Asseler is with the Department of Nuclear Medicine, Ghent University Hospital, De Pintelaan 185, B-9000 Ghent, Belgium.

I. Lemahieu is with the Department of Electronics and Information Systems, MEDISIP, Ghent University-IBBT-IBiTech, B-9000 Ghent, Belgium.

Color versions of one or more of the figures in this paper are available online at http://ieeexplore.ieee.org.

Digital Object Identifier 10.1109/TMI.2008.923698

\section{INTRODUCTION}

$\mathbf{D}^{2}$ YNAMIC positron emission tomography (PET) is a molecular imaging technique that is used to monitor the in vivo spatio-temporal distribution of a radiolabeled tracer. Dynamic imaging provides richer information than conventional static PET imaging and has the ability to generate quantitative information about physiological parameters through the identification of kinetic or compartmental models.

PET data are collected as projections in sinograms or listmode format. Tomographic reconstruction is required to obtain the spatial distribution of the radioactive tracer from these indirect measurements. Dynamic PET reconstruction is challenging due to the small number of accumulated counts in each time-bin. The standard paradigm is to constrain the solution using a regularization term, which acts as an implicit image model, and makes the inverse problem well-conditioned [2], [3]. Various flavors of regularization have been deployed for the reconstruction of static images [4]-[6]. Some of these methods have been extended to perform spatio-temporal reconstructions [7]-[9].

A number of multiscale and wavelet-based methods have also been proposed for solving inverse problems, the rationale being that wavelets are very good at preserving edges. The simplest among these are wavelet-based postprocessing techniques, which have been successfully applied by several groups in the context of dynamic PET reconstructions [10]-[12]; these typically yield significant increases in signal-to-noise ratio (SNR) without sacrificing spatio-temporal resolution. Wavelets have also been incorporated into the reconstruction process itself. Prominent examples are the wavelet-vaguelette [13], [14] and vaguelette-wavelet [15] decomposition followed by thresholding of the coefficients [16]. More recently, wavelet-based regularization methods have been developed for solving inverse problems including deconvolution [17], [18]. There is also a connection between wavelets and some recent graph models that have been developed for Bayesian tomographic reconstruction from Poisson data [19], [20].

The more recent class of methods is based upon the idea of imposing a sparsity constraint by penalizing the $\ell_{1}$-norm of the wavelet coefficients $\boldsymbol{w}$ : this leads to a criterion of the form (see [1])

$$
\lambda\left(x_{1}, x_{2}\right)=\arg \min _{\lambda \geq 0}\left\{\left\|\boldsymbol{y}-\mathrm{P} \lambda\left(x_{1}, x_{2}\right)\right\|_{2}^{2}+\mu\|\boldsymbol{w}\|_{1}\right\}
$$

where the imaging operator $\mathrm{P}$ expresses the forward model from the spatial object $\lambda\left(x_{1}, x_{2}\right)$ to the observations $\boldsymbol{y}$, where $\mu$ is the tuning (or regularization) parameter, and where $\boldsymbol{w}$ denotes the 2-D wavelet transform of the object $\lambda\left(x_{1}, x_{2}\right)$. The use of 
the $\ell_{1}$-term for the regularization favors a sparse wavelet representation with the advantage that the functional is convex. Another interesting aspect is that this criterion can be optimized using the iterative thresholding algorithm of Daubechies et al. [1]. In this paper, we extend the nonparametric static reconstruction that would follow from (1) to a dynamic (spatio-temporal) setting. Moreover, instead of using conventional wavelets in the temporal dimension, we opt for $E$-spline wavelets [21] that are better matched to the type of responses encountered in dynamic PET studies. The key concept is that the activity distribution in the body is ruled by a system of differential equations involving compartmental models. The proposed $E$-spline wavelets are ideally suited for the sparse representation of solutions of these differential equations.

While we are not aware of any work in this area involving wavelets, many researchers have shown the advantage of using tailored temporal basis functions for the representation of TACs in dynamic PET reconstruction. Snyder [22] proposed to use rate functions described by convolutions of the input function with a basis of exponential functions. Several teams have investigated the use of nonuniform $B$-splines and have reported promising results [7]-[9], [23]. It has also been proposed to derive the temporal basis functions from the data itself [24], [25]. Our work differs from these approaches in that the proposed temporal basis functions form a (generalized) wavelet basis and in that we make use of a global $\ell_{1}$ sparsity constraint in the spatio-temporal wavelet domain.

The paper is organized as follows. In Section II, we briefly review PET image formation and the wavelet image representation. In Section III-A, we discuss the key properties of the introduced $E$-spline wavelets. In Section III-B, we review the primary compartmental models that have been proposed for modeling PET time activity curves. We show that the time course can be obtained as the solution of a differential equation which provides us with an explicit link with the $E$-spline wavelet representation. In Sections III-C and III-D, we discuss and illustrate the selection of $E$-spline wavelet parameters (poles and zeros) in a realistic simulation. In Section IV, we address some implementation issues concerning the system model, the reconstruction algorithm, the prefilter and tuning of the threshold parameter. Finally, in Sections V and VI, we perform an evaluation of the $\ell_{1}$ regularization and the $E$-spline wavelets using 1-D and 3-D computer simulations and illustrate the application of the method to two clinical data sets.

\section{SPATIO-TEMPORAL ReCONSTRUCTION PROBLEM FORMULATION}

\section{A. Image Formation}

We consider the problem of reconstructing a (nonnegative) spatio-temporal activity distribution $\lambda\left(x_{1}, x_{2}, \tau\right)$ from dynamic PET data $\boldsymbol{y}=\mathrm{P} \lambda$ [2], [3]. The basic physical model of the spatially-variant imaging operator ${ }^{1} \mathrm{P}$ is a spatially-weighted integration of $\lambda\left(x_{1}, x_{2}, \tau\right)$

$$
\bar{y}_{b}(\tau)=\iint_{\Omega} \lambda\left(x_{1}, x_{2}, \tau\right) h_{b}\left(x_{1}, x_{2}\right) d x_{1} d x_{2}
$$

\footnotetext{
${ }^{1}$ In the current setting, we assume a temporally-invariant system model $\mathrm{P}$ corresponding to a full-ring detector with no rotating parts as usually encountered in PET systems.
}

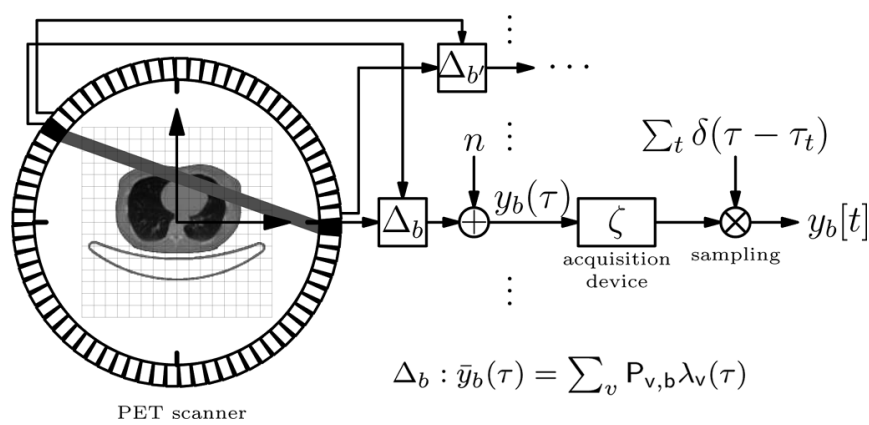

Fig. 1. Diagram representation of the data acquisition. Forward model $\mathrm{P}$ is illustrated by the gray tube, coincidence bin $b$ is formed by the black detector crystals. Noise term $n$ is added to the data that are collected in a number of shorter time frames indexed by $t$ and $\tau_{t}$ corresponds to the start time of frame $t$. General sampling consists of a temporal integration $(\zeta)$ followed by a sampling $\sum_{t} \delta\left(\tau-\tau_{t}\right)$.

where $h_{b}\left(x_{1}, x_{2}\right)$ represents the tube-shaped sensitivity profile of the coincidence detector $b$. The discretized version reads $\bar{y}_{b}(\tau)=\sum_{v} \mathrm{P}_{v, b} \lambda_{v}(\tau)$, where the sum is over all pixels $v$. At coincidence in bin $b$, we observe an inhomogeneous Poisson process with rate density $\bar{y}_{b}(\tau)$.

The sinogram is acquired progressively by collecting data in a number of short time frames. In the present setting, all time frames are $\Delta \tau$ long. The general data sampling is therefore modelled as a temporal integration at the coincidence bins

$$
y_{b}[t]=\int y_{b}(\tau) \zeta\left(\tau-\tau_{t}\right) d \tau=\left\langle y_{b}(\tau), \zeta\left(\tau-\tau_{t}\right)\right\rangle
$$

where $y_{b}[t]$ denotes the number of detected counts in bin $b$ during the $t$ th time frame, $\tau_{t}$ corresponds to the start time of frame $t, \zeta(\tau)=1$ if $\tau \in[0, \Delta \tau)$ and zero elsewhere. The noise is conceptually modelled as an additive noise term $y_{b}(\tau)=\bar{y}_{b}(\tau)+n(\tau)$. The acquisition process is illustrated in Fig. 1 .

By convention we define one time-unit as $\Delta \tau$ so that we consider integer frame start times: $\tau_{t} \in \mathbb{Z}$.

\section{B. Wavelet Regularized Formulation}

Denoting $\boldsymbol{w}$ as the spatio-temporal wavelet transform of $\lambda$, the regularized-reconstruction task is thus to find the nonnegative minimizer $\hat{\lambda}$ of the criterion

$$
\left\|\boldsymbol{y}-\mathrm{P} \lambda\left(x_{1}, x_{2}, \tau\right)\right\|_{2}^{2}+\mu\|\boldsymbol{w}\|_{1} .
$$

The first term is the data term and is given by the difference between the projections of the reconstruction $\lambda\left(x_{1}, x_{2}, \tau\right)$ and the measured projections $\boldsymbol{y}$. This least-squares term can be associated to a Gaussian noise model. The second term is a penalty that stabilizes the reconstruction. It is given by the $\ell_{1}$-norm of the (spatio-temporal) wavelet coefficients $\boldsymbol{w}$ of $\lambda$ which favours a sparse description of the image in the wavelet domain. The tuning parameter $\mu$ controls the data-fit versus regularity tradeoff. The key difference with (1) is that we have extended the optimization over the temporal dimension, which is potentially advantageous for noise reduction, especially when there is a strong correlation within TACs. 
The main contribution of this paper is our special treatment of spatio-temporal regularization. This is achieved by using different types of wavelets in space and time, respectively. This approach is inspired from the fact that the spatial and temporal characteristics of the image are very different. In the spatial domain, we use $B$-spline (Battle-Lemarié) wavelets that have been extensively used in (biomedical) imaging (e.g., [26], [27]). In the temporal domain, we are introducing $E$-spline wavelets. This methodology can be easily implemented by considering a separable wavelet basis. Using a tailored wavelet basis will result in a much sparser representation of the image. The sparse description of the true image in the wavelet domain is an important prerequisite to the success of the criterion (4); indeed, the basic motivation for using the proposed $\ell_{1}$ penalty is that it is a good proxy for the $\ell_{0}$-norm, which is a direct measure of sparsity but which has the disadvantage of being nonconvex. It has been proven that this works well provided that the true signal is sufficiently sparse [28].

Formally we represent the activity distribution $\lambda\left(x_{1}, x_{2}, \tau\right)$ by an expansion onto an orthogonal wavelet basis [29], [30]

$$
\lambda\left(x_{1}, x_{2}, \tau\right)=\sum_{\boldsymbol{j}} \sum_{\boldsymbol{k}} w_{\boldsymbol{j}}[\boldsymbol{k}] \psi_{\boldsymbol{j}, \boldsymbol{k}}\left(x_{1}, x_{2}, \tau\right)
$$

where $\boldsymbol{j}=\left(j_{1}, j_{2}, j_{3}\right)$ and $\boldsymbol{k}=\left(k_{1}, k_{2}, k_{3}\right)$ are the scale and translation parameter vectors, $w_{j}[k]$ the wavelet coefficients and $\psi_{\boldsymbol{j}, \boldsymbol{k}}\left(x_{1}, x_{2}, \tau\right)$ the basis functions which are either wavelets or scaling functions. We use wavelets that are separable in space and time

$$
\psi_{\boldsymbol{j}, \boldsymbol{k}}\left(x_{1}, x_{2}, \tau\right)=\psi_{j_{1}, k_{1}}^{(1)}\left(x_{1}\right) \psi_{j_{2}, k_{2}}^{(1)}\left(x_{2}\right) \psi_{j_{3}, k_{3}}^{(2)}(\tau)
$$

where $\left\{\psi_{j, k}^{(\cdot)}\right\}_{j=1, k \in \mathbb{Z}}^{j_{\max }}$ are the 1-D continuously-defined wavelets; the subscript (1) and (2) denote $B$-spline and $E$-spline wavelets, respectively. Note that we use the same wavelets $\left(\psi_{j, k}^{(1)}(x)\right)$ for the two spatial dimensions.

In the next sections, we will present the link between the $E$-spline defining differential operator $\mathrm{L}_{\alpha \gamma}[21]$ and the system of differential equations that rules the PET signal.

\section{Temporal Model Selection}

\section{A. E-Spline Wavelets}

$E$-spline wavelets are a generalization of $B$-spline wavelets and are specified by the exponential parameter vectors $\boldsymbol{\alpha}$ (poles) and $\boldsymbol{\gamma}$ (zeros). The dimension of vector $\boldsymbol{\alpha}$ is $N$ and the vector consists of $N_{d}$ distinct $\alpha_{l}$ 's of multiplicity $\left\{m_{l}\right\}_{1}^{N_{d}}$ with $N=$ $\sum_{l=1}^{N_{d}} m_{l}$. The dimension of $\gamma$ is $M$ with $M<N$. The interest of using $E$-spline wavelets is that they generalize the vanishing moments property of the conventional wavelets. Let $\psi_{j, k}^{(2)}(\tau)$ be an $E$-spline wavelet defined by $\boldsymbol{\alpha}$ and $\boldsymbol{\gamma}$ then we have that [21], [31], [32]

$$
\int_{-\infty}^{+\infty} \tau^{n} e^{\alpha_{l} \tau} \psi_{j, k}^{(2)}\left(\tau-\tau_{0}\right) d \tau=0, \quad \forall \tau_{0} \in \mathbb{R}, n=0, \ldots, m_{l}-1 .
$$

We say that $\psi_{j, k}^{(2)}$ has $N$ vanishing exponential moments. This implies that the $E$-spline wavelets are able to kill all exponential signals of the form

$$
p_{\boldsymbol{\alpha}}(\tau)=\sum_{l=1}^{N_{d}} \sum_{n=0}^{m_{l}-1} c_{l} \tau^{n} e^{\alpha_{l} \tau}
$$

where $c_{l}$ are arbitrary coefficients. This property of the $E$-spline wavelets translates into a sparse representation of piecewise exponential signals as many wavelet coefficients vanish. A sparse description of the signal is an important prerequisite to the success of the $\ell_{1}$ penalty. Note that we recover the $B$-spline wavelets of degree $N-1$ if $\boldsymbol{\gamma}=\{\}$ and all $\alpha$ 's are 0 [21]. Further details are discussed in Appendix.

The corresponding $E$-spline function-space is a generalization of the classical polynomial splines. The $E$-spline specified by $\boldsymbol{\alpha}, \boldsymbol{\gamma}$ and knots $-\infty<\cdots<\tau_{t}<\tau_{t+1}<\cdots<+\infty$ is a function that contains discontinuities of order $N$ at the knot points $\tau_{t}$ but that is very smooth otherwise [31]. Formally, the $E$-spline function-space is given by [31]

$$
\mathcal{V}_{0}^{(2)}=\left\{f: \mathrm{L}_{\boldsymbol{\alpha}, \gamma}\{f(\tau)\}=\sum_{t \in \mathbb{Z}} c_{t} \delta\left(\tau-\tau_{t}\right)\right\} \cap L_{2}(\mathbb{R})
$$

where the underlying linear differential operator $\mathrm{L}_{\alpha, \gamma}$ is defined in the Laplace domain as

$$
\mathrm{L}_{\boldsymbol{\alpha}, \boldsymbol{\gamma}}(s)=\frac{\prod_{n=1}^{N}\left(s-\alpha_{n}\right)}{\prod_{m=1}^{M}\left(s-\gamma_{m}\right)} .
$$

The operator corresponds to the linear differential system $\mathrm{L}_{\boldsymbol{\alpha}, \boldsymbol{\gamma}}(s) F(s)=G(s)$

$$
\begin{aligned}
& D^{N} f(\tau)+a_{N-1} D^{N-1} f(\tau)+\cdots+a_{0} f(\tau) \\
& \quad=D^{M} g(\tau)+b_{M-1} D^{M-1} g(\tau)+\cdots+b_{0} g(\tau)
\end{aligned}
$$

where $D^{n}=d^{n} / d \tau^{n}$ is the $n$ th-order derivative, $\boldsymbol{\alpha}$ and $\boldsymbol{\gamma}$ correspond to the roots of the polynomials $s^{N}+a_{N-1} s^{N-1}+\cdots+a_{0}$ and $s^{M}+b_{M-1} s^{M-1}+\cdots+b_{0}$, respectively. Note that if $\gamma=\{\}$ and all $\alpha$ 's are 0 the function-space corresponds to the polynomial splines (which we will denote as $\mathcal{V}_{0}^{(1)}$ ).

In the right part of Fig. 2, some $E$-splines are illustrated. From this figure and (9), an alternative interpretation of the $E$-spline wavelets' ability to generate a sparse representation of exponential signals is apparent. Clearly, if there are only few $c_{t} \neq 0$ then $\mathrm{L}_{\boldsymbol{\alpha}, \boldsymbol{\gamma}}\{f(\tau)\}$ generates a sparse description of the exponential signal. In [21] it is shown that the $E$-spline wavelets $\psi_{j, k}^{(2)}$ essentially behave as multiscale versions of the underlying operator $\mathrm{L}_{\boldsymbol{\alpha}, \boldsymbol{\gamma}}$ (see also Appendix A) thus generating a sparse wavelet representation of $f(\tau)$.

\section{B. Compartmental Models for PET}

The motivation to use $E$-spline wavelets for the representation of the temporal variations of the radiotracer is that we can interpret the TACs as $E$-splines. To show this, we need to identify the underlying differential operator. The left part of Fig. 2 illustrates how the operator $\mathrm{L}_{\boldsymbol{\alpha}, \boldsymbol{\gamma}}$ is identified. The pulsetrain of 


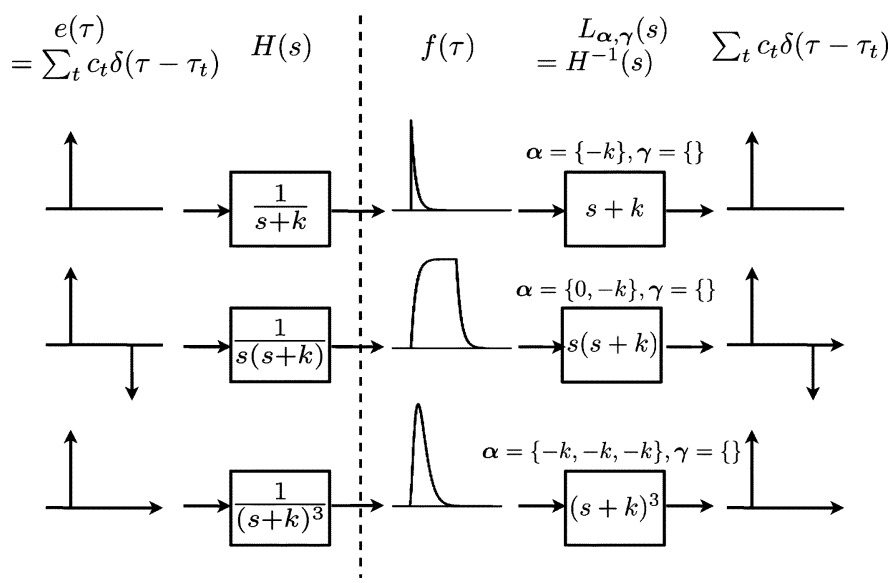

Fig. 2. Typical exponential splines $f(\tau)$ encountered in dynamic PET. Top row: time course of the intraveneous bolus injection; middle: infusion; bottom: typical plasma activity concentration. Illustration of the link between the transfer function $H(s)$ and the differential operator $\mathrm{L}_{\boldsymbol{\alpha}, \boldsymbol{\gamma}}(s)$. Left: generation of the time curves as a response to short injection events. Right: analysis of the time curves with the operator $\mathrm{L}_{\boldsymbol{\alpha}, \boldsymbol{\gamma}}$.

stimulations $e(\tau)$ on the left side of the figure represents short events that correspond to the tracer injections; when the Laplace transform $\mathrm{L}_{\boldsymbol{\alpha}, \gamma}(s)$ of the spline-defining operator is matched to the inverse of the transfer function $H(s)$ of the system of differential equations that generates the PET signal, then applying $\mathrm{L}_{\boldsymbol{\alpha}, \boldsymbol{\gamma}}$ yields back the pulses. The $E$-spline wavelet transform is able to approximately replicate this behavior if the parameters are chosen appropriately. The relevant transfer function can be identified from the compartmental model description of the PET signal [33]-[36].

1) PET Signal: The best known kinetic model is probably the two-tissue compartmental model that describes the uptake of $\left[{ }^{18} \mathrm{~F}\right]$ - FDG [37], [38]. The system takes the blood plasma activity as input and produces an output that corresponds to the PET signal. We consider here a general time-invariant linear tissue model that consists of $n$ tissue compartments [34]. The system is conveniently described using a state space representation

$$
\begin{aligned}
& \dot{\boldsymbol{x}}(\tau)=\mathrm{A} \boldsymbol{x}(\tau)+\mathrm{B} \boldsymbol{u}(\tau) \\
& \boldsymbol{y}(\tau)=\mathrm{C} \boldsymbol{x}(\tau)+\mathrm{D} \boldsymbol{u}(\tau) \\
& \boldsymbol{x}(0)=\boldsymbol{x}_{0}
\end{aligned}
$$

where the $\boldsymbol{x}(t)$ is the $(n \times 1)$ vector of state variables, $\dot{\boldsymbol{x}}(t)$ denotes the first derivative of $x(t), \mathrm{A}$ is the $(n \times n)$ transition matrix, $\mathrm{B}$ is the $(n \times m)$ input matrix, $\boldsymbol{u}(t)$ is the $(m \times 1)$ input vector, $\boldsymbol{y}(\tau)$ is the $(l \times 1)$ observation vector, $\mathrm{C}$ is the $(l \times n)$ observation matrix, $\mathrm{D}$ is the $(l \times m)$ feedforward matrix, and $\boldsymbol{x}_{0}$ is the $(n \times 1)$ vector with the initial conditions.

In our case, the state variables are the activity concentrations in the $n$ individual tissue compartments and A contains the rate coefficients that describe the activity exchange between the individual compartments. The input consists of two elements $(m=$ 2 ): the (unmetabolized) activity in the plasma $u_{1}(\tau)=u_{p l}(\tau)$ and in the whole blood $u_{2}(\tau)=u_{w b}(\tau)$, respectively. The observation $y_{1}(\tau)=y(\tau)$ is the PET signal $(l=1)$ and we assume that there was no activity present before the first injection: $\boldsymbol{x}_{0}=\mathbf{0}$. In general, the activity exchange between the blood and the tissue is regulated by one single coupling between the plasma activity $u_{p l}(\tau)$ and one individual tissue compartment $x_{1}(\tau)$. This results in an input matrix $\mathrm{B}=\left(k_{1} \boldsymbol{e}_{1} \mid \mathbf{0}\right)$, where $\boldsymbol{e}_{1}$ is the $(n \times 1)$ unit vector in the $x_{1}$-direction.

The PET signal is composed of the total activity in the tissue $y_{\text {tis }}(\tau)$ plus a contribution $y_{\text {vas }}(\tau)$ from the vasculature $u_{w b}(\tau)$. We can therefore write $C$ as $\left(1-V_{B}\right) 1^{T}$ and $\mathrm{D}$ as $\left(0 V_{B}\right)$, where $V_{B}$ is the fractional blood volume and with 1 is a $(n \times 1)$ vector of ones. Using the Laplace transform, we have that

$$
\begin{aligned}
Y_{\mathrm{tis}}(s) & =H_{1}(s) U_{p l}(s) \\
& =\left(1-V_{B}\right) \mathbf{1}^{T}(s \mathbf{I}-\mathrm{A})^{-1} k_{1} \boldsymbol{e}_{1} U_{p l}(s) \\
Y_{\text {vas }}(s) & =H_{2}(s) U_{w b}(s) \\
& =V_{B} U_{w b}(s) .
\end{aligned}
$$

2) Plasma Activity: Compartmental models that transform the time course of the activity measured in whole blood into the time course of the radiotracer in plasma are given in [34] and [39]. A similar state space analysis can be performed and we have that $U_{p l}(s)=H_{3}(s) U_{w b}(s)$.

3) Whole Blood Activity: Models describing the whole blood activity have also been discussed [40]-[42]. These models take the intravenous injected tracer concentration $u_{\text {inj }}(\tau)$ and transform it into a whole blood activity signal. We have formally that $U_{w b}(s)=H_{4}(s) U_{\text {inj }}(s)$. It should be noted that the gammavariate that has often been used to fit the time course of the blood activity functions [43], [44] can also be formulated as a solution to a compartmental model [40].

4) Intravenous Injection: Differential models for the time course of the intravenous injection for bolus and constant infusion have been described in [42]. The simplest model for a bolus is an instantaneous mixing in the plasma compartment. More realistic models include one compartment [42] and we can write $U_{\text {inj }}(s)=H_{5}(s) E(s)$, where $E(s)$ is the Laplace transform of the events $e(\tau)=\sum_{k} c_{t} \delta\left(\tau-\tau_{t}\right)$. The models for bolus and infusion injections are illustrated in the upper two rows of Fig. 2.

5) Total System: Specifically, the global effect is that of a cascade of systems transforming the injection events $e(\tau)$ into the PET signal $y(\tau)$ as depicted in Fig. 3. We have that $Y(s)=H_{\text {tot }}(s) E(s)$, with $H_{\text {tot }}(s)=\left[H_{2}(s)+H_{1}(s) H_{3}(s)\right] H_{4}(s) H_{5}(s)$. Thus, we may interpret the PET signal $y(\tau)$ as an $E$-spline associated with the differential operator for which holds $\mathrm{L}_{\boldsymbol{\alpha}, \boldsymbol{\gamma}}(s)=H_{\text {tot }}^{-1}(s)$

$$
\mathrm{L}_{\boldsymbol{\alpha}, \boldsymbol{\gamma}}\{y(\tau)\}=e(\tau)=\sum_{t} c_{t} \delta\left(\tau-\tau_{t}\right)
$$

where $\tau_{t}$ represent the stimulation times. The parameter vectors $\boldsymbol{\alpha}$ and $\boldsymbol{\gamma}$ are obtained as the poles and zeros of $H_{\text {tot }}(s)$, respectively.

Note that we assume that the stimulation times of the pulsetrain $e(\tau)$ correspond to the start times $\tau_{t}$ of the acquired time frames. This approximation is appropriate if we choose the timeunit $\Delta \tau$ small enough.

\section{Selection of E-Spline Wavelet Parameters}

When designing the temporal $E$-spline wavelets $\psi^{(2)}(\tau)$ for the PET reconstructions we need to specify the parameter vectors $\boldsymbol{\alpha}$ and $\boldsymbol{\gamma}$. However, the poles and zeros of the transfer function $H(s)$ clearly depend on the rate coefficients that describe 


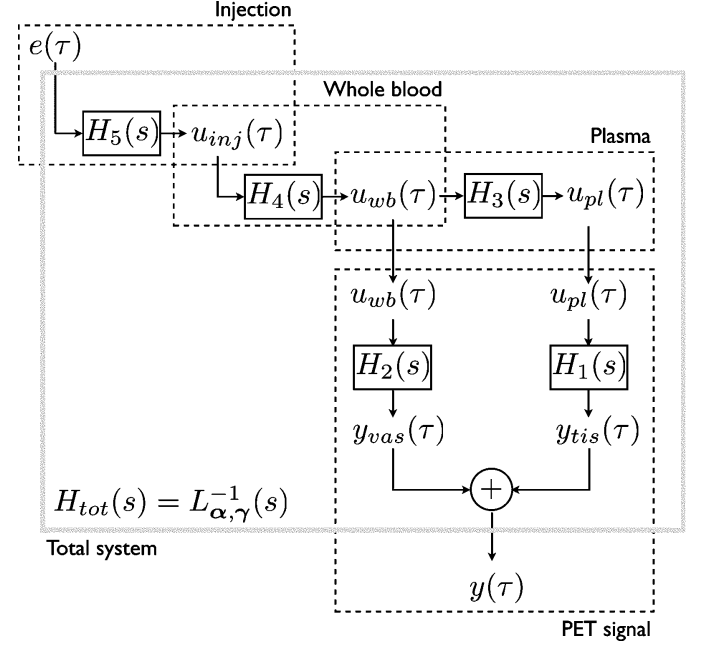

Fig. 3. Schematic representation of the cascade of systems transforming the injection events $e(\tau)$ into the PET signal $y(\tau)$.

the exchange between the compartments. Unfortunately, most tissue rate coefficients are unknown. In fact, the dynamic PET experiments are being designed to estimate those coefficients. In other words the transfer function $H_{1}(s)$ is unknown. Moreover, when developing models for newly-developed tracers the number of compartments $n$ in tissue, thus the order of $H_{1}(s)$, is unknown. Assigning the exact $\alpha$ and $\gamma$ prior to the PET reconstruction is thus impossible. Furthermore, different regions in the image are described by different kinetic parameters.

The time courses of the whole blood and plasma activity, on the other hand, are generally obtained from blood samples. From these measurements the transfer functions $H_{3}(s), H_{4}(s)$, and $H_{5}(s)$ can be approximated.

In designing the multiresolution decomposition of the signal, we have built in a certain robustness against the selection of $\boldsymbol{\alpha}$ and $\boldsymbol{\gamma}$. However, the more accurately the parameters $\boldsymbol{\alpha}$ and $\boldsymbol{\gamma}$ are chosen, the sparser the signal can be represented, which is advantageous for the $\ell_{1}$ regularization.

Therefore, we select a $\boldsymbol{\alpha}$ and $\boldsymbol{\gamma}$ vectors that approximately capture the important properties of transfer function $H(s)$. The appropriate selection of $\boldsymbol{\alpha}$ and $\boldsymbol{\gamma}$ is a study-dependent process. As a general rule, we recommend to avoid the use of high orders $N$ so that the constructed wavelets are reasonably localized. In all our experiments, we have used $\boldsymbol{\alpha}$ vectors with $N=3$. The $\boldsymbol{\alpha}$ vector should contain at least one 0 , so that the $E$-spline wavelets are able to reconstruct the baseline. Other $\alpha$ 's are chosen to model the high frequency behavior. In our experiments we have used one additional $\alpha$ of multiplicity 2 which approximately modeled the highest frequency we expected in our data. An important observation is that the tissue signal will not contain higher frequency components than the whole blood and plasma signal and that the whole blood activity will play an important role in the selection of the high frequency characteristics of the parameter vectors. Finally, in all our experiments, we have set $\boldsymbol{\gamma}=\{\}$ and we did not investigate the influence of $\boldsymbol{\gamma}$. A specific example of parameter selection is considered next. The robustness of the method with respect to parameter variations will be illustrated in the examples Section VI.

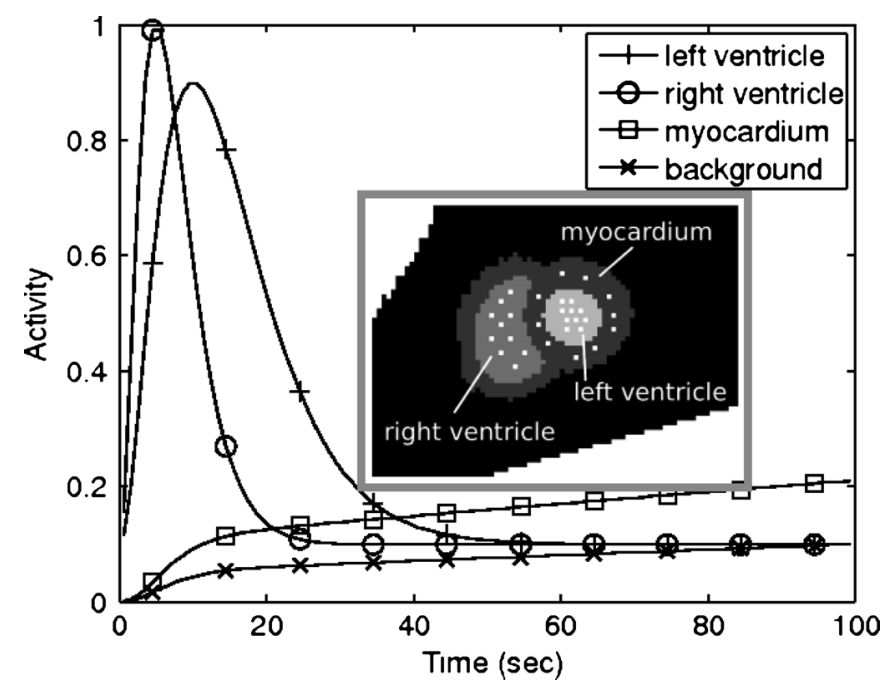

Fig. 4. Cardiac $\left[{ }^{13} \mathrm{~N}\right]-\mathrm{NH}_{3}$ imaging simulation experiment. TACs for the different regions and slice of the NCAT phantom $(31 \mathrm{~cm} \times 25 \mathrm{~cm})$ with the positions of the pixels used for the analysis.

\section{Example: The $\left[{ }^{13} \mathrm{~N}\right]-\mathrm{NH}_{3}$ Kinetic Model}

We illustrate the parameter selection for a myocardial perfusion study using $\left[{ }^{13} \mathrm{~N}\right]$-Ammonia PET. The corresponding spatial distribution map and time activity curves are shown in Fig. 4.

This case is challenging as the image contains regions of pure whole blood signal (the heart chambers) and tissue regions where the transfer functions $H(s)$ may be quite different. Furthermore, myocardial perfusion studies are often performed without arterial blood sampling. The time course of the left ventricular activity is then taken as the blood input function for the fitting of the tissue model to the data $\left(H_{3}(s)=1\right)$. Thus, neither $H_{1}(s), H_{4}(s)$ nor $H_{5}(s)$ are known prior to the reconstruction.

1) Whole Blood: The whole blood signal is well modelled by [43]

$$
\lambda(\tau)=d_{1} \tau^{a} e^{-b \tau}+d_{2}\left(1-e^{-c \tau}\right)
$$

with parameters $a$ (between 2-9), $b$ (between 0.5-0.11 $\left(s^{-1}\right)$ ), $c$ is reasonable small (no values given in [43]), and $d_{1}$ is scaled so that the maximum of $\lambda(\tau)$ is approximately $10 d_{2}$. The first term is a gamma variate, the second term is the recirculation term.

In our case, we consider the TAC model $5 \tau^{2} e^{-0.4 \tau}+2(1-$ $\left.e^{-0.5 \tau}\right)$ which corresponds to the impulse response of the system with transfer function

$$
H_{a}(s)=\frac{(s+10.69)(s+0.5002)(s+0.01197)}{s(s+0.5)(s+0.4)^{3}} .
$$

In a first approximation, we cancel the terms $(s+0.5002) /(s+$ $0.5)$ and $(s+0.01197) / s$, and approximate $s+10.69$ by a constant in the frequency interval of interest. Thus, we select the parameter vectors $\boldsymbol{\alpha}_{1}=\{-0.4,-0.4,-0.4\}$ and $\boldsymbol{\gamma}=\{\}$.

2) Tissue Response: The tissue model is described by a two compartmental model [43]. If we consider a pure tissue signal, i.e., $V_{B}=0$, then the transfer function for the tissue response is given by

$$
H_{b}(s)=\frac{s+b}{s(s+a)}
$$


TABLE I

TYPICAL VALUES FOR THE PARAMETERS OF THE $\left[{ }^{13} \mathrm{~N}\right]-\mathrm{NH}_{3}$ TISSUE RESPONSE

\begin{tabular}{|c|c|c||c|c|c|}
\hline$F$ & $a$ & $b$ & $F$ & $a$ & $b$ \\
\hline \hline 0.2 & 3.559 & 3.554 & 2.5 & 0.1416 & 0.08949 \\
\hline 0.5 & 0.2092 & 0.1988 & 4 & 0.1878 & 0.1045 \\
\hline 1 & 0.1199 & 0.09905 & 5 & 0.2205 & 0.1164 \\
\hline
\end{tabular}

where $a$ and $b$ are parameters that depend on the rate constants of the model such as the myocardial flow $F$. Typical values for $a$ and $b$ as found in humans [43] are given in Table I.

For low flow $(F)$ values it is easy to see that the system is well approximated by an integrator $(1 / s)$. For higher flow values, we can neglect the effect of $a$ and $b$ and the system is still reasonably well approximated by an integrator $(1 / s)$. This is illustrated in Fig. 5 by the amplitude diagram of the transfer function $H_{b}(s)$ and by the impulse responses of $H_{a}(s) H_{b}(s)$ for different values of $F$. This system requires the parameter vector $\boldsymbol{\alpha}_{2}=\{0\}$ and $\boldsymbol{\gamma}=\{\}$.

3) Total System: The transfer function for the global system is easily identified. We have that $H_{4}(s) H_{5}(s)=H_{a}(s)$, $H_{1}(s) H_{3}(s)=\left(1-V_{B}\right) H_{b}(s)$, and $H_{2}(s)=1$. In the image, there are regions of almost pure blood $\left(V_{B} \approx 1\right)$ and of almost pure tissue $\left(V_{B} \approx 0\right)$. Therefore, the parameter vectors $\boldsymbol{\alpha}=\left(\boldsymbol{\alpha}_{1} \mid \boldsymbol{\alpha}_{2}\right)=\{0,-0.4,-0.4,-0.4\}$ and $\gamma=\{\}$ form a good compromise for the modelling task.

\section{IMPLEMENTATION}

\section{A. System Model}

The projector for the reconstruction $\mathrm{P}$ was based on the linelength model and involved multiple tracings per detector pair. We have considered a 2-D system model. The clinical data were therefore first rebinned into 2-D slices using the single slice rebinning algorithm. The projector did not consider an attenuation model. The clinical data were precorrected for attenuation while the synthetic experiments did not include photon attenuation.

\section{B. Reconstruction Algorithm}

To find the minimizer of (4), we use the iterative thresholding algorithm as proposed in [1], which computes the next estimate of the image as

$$
\lambda^{n+1}=\mathcal{N} \mathrm{S}_{\mu}\left(\lambda^{n}+\mathrm{P}^{T}\left(y-\mathrm{P} \lambda^{n}\right)\right), \quad n=0,1, \ldots,
$$

where $n$ is the iteration number, $\mathcal{N}$ is the projector on the nonnegative functions (see Section IV-C), $\mu$ is the tuning parameter [see (4)], and the nonlinear operator $S_{\mu}$ acts in the wavelet domain coefficient-wise

$$
\mathrm{S}_{\mu}(g)=\sum_{\boldsymbol{j}, \boldsymbol{k}} \mathcal{S}_{\mu}\left(w_{\boldsymbol{j}}[k]\right) \psi_{\boldsymbol{j}, \boldsymbol{k}}=\sum_{\boldsymbol{j}, \boldsymbol{k}} \mathcal{S}_{\mu}\left(\left\langle g, \psi_{\boldsymbol{j}, \boldsymbol{k}}\right\rangle\right) \psi_{\boldsymbol{j}, \boldsymbol{k}}
$$

where $\mathcal{S}_{\mu}$ is the soft-thresholding function

$$
\mathcal{S}_{\mu}(x)=\left\{\begin{array}{ll}
x+\frac{\mu}{2}, & \text { if } x \leq-\frac{\mu}{2} \\
0, & \text { if }|x|<\frac{\mu}{2} \\
x-\frac{\mu}{2}, & \text { if } x \geq \frac{\mu}{2}
\end{array} .\right.
$$

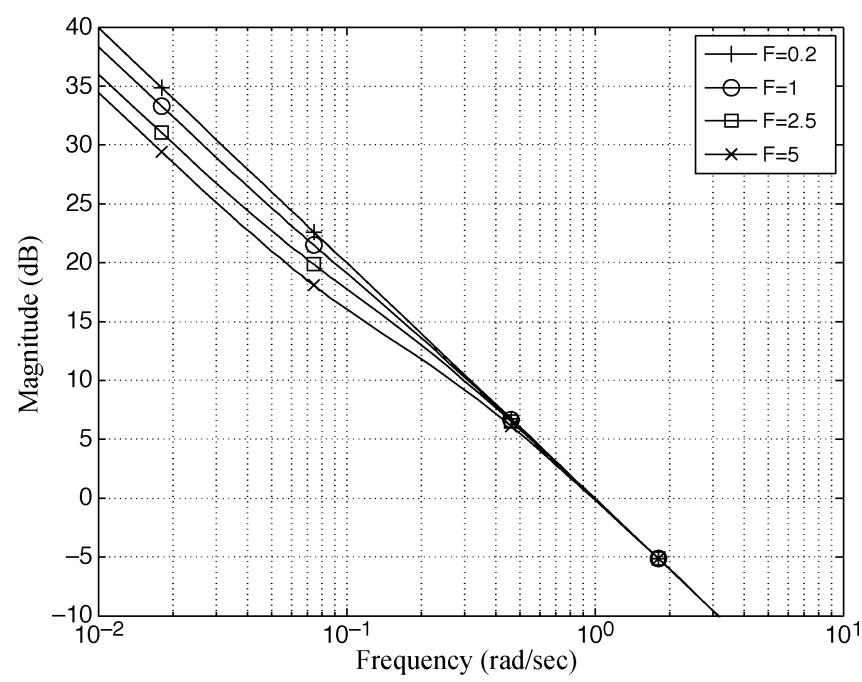

(a)

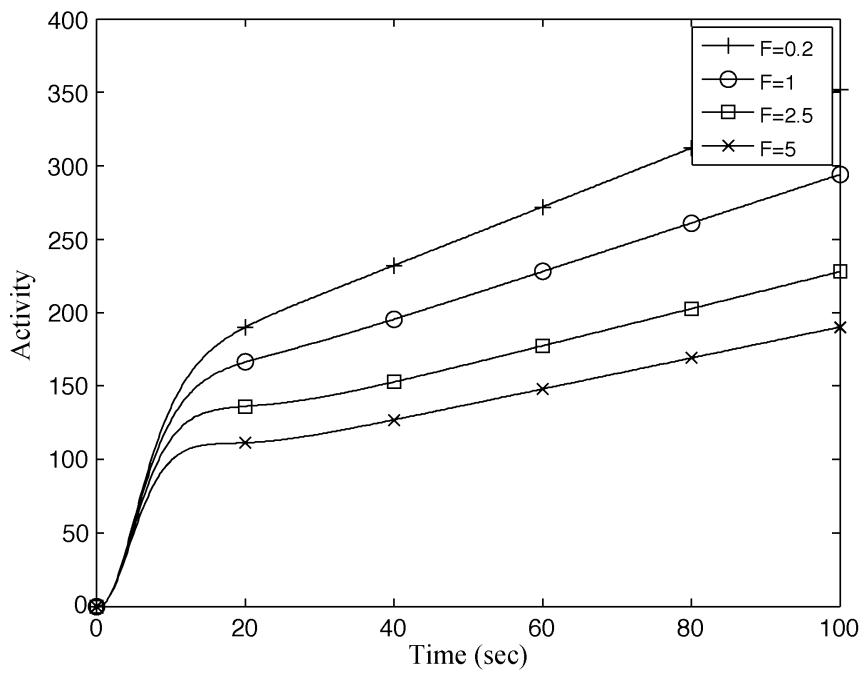

(b)

Fig. 5. $E$-spline parameter selection example. (a) Amplitude diagram of $H_{b}(s)$ for a range of flow values $(F)$. For low values of $F$ the amplitude characteristic almost coincides with the amplitude characteristic of $1 / s$ (line of slope $-20 \mathrm{~dB} /$ decade). (b) Impulse response of the total tissue response $H_{a}(s) H_{b}(s)$. Qualitatively the curves corresponds to an integration after a low-pass filtering. Impulse response with $H_{b}(s)=1 / s$ (not shown) is very close to the curve for $F=0.2$.

One iteration nicely decomposes into three steps: an unregularized update of the estimate followed by a denoising step [45], [46] and a projection onto the nonnegative solutions. The denoising step (22) involves a wavelet analysis step $\left(\left\langle g, \psi_{\boldsymbol{j}, \boldsymbol{k}}\right\rangle\right)$, a soft thresholding $\left(\mathcal{S}_{\mu}(x)\right)$, and a wavelet synthesis step. In our experiments, we have used a zero start image $\lambda^{0}=\mathbf{0}$.

\section{Approximation in $\mathcal{V}_{0}$}

By including a full range of scales and spatio-temporal translations $\left(k_{1}, k_{2}, k_{3}\right) \in \mathbb{Z}^{3}$ in (5), the wavelet functions can be specified to form an orthonormal basis for the Hilbert space of square integrable functions $L_{2}\left(\mathbb{R}^{2} \times[0, T]\right)$. In practice, we consider an approximation space $\mathcal{V}_{0}=\mathcal{V}_{0}^{(1)} \times \mathcal{V}_{0}^{(1)} \times \mathcal{V}_{0}^{(2)} \subset L_{2}\left(\mathbb{R}^{2} \times[0, T]\right)$, where 
$\mathcal{V}_{0}^{(\cdot)}$ is defined in Section III-A. The function-space is spanned by the finest-scale scaling functions $\phi_{0, k}^{(\cdot)}$ [29], [30]. The approximation is done by only considering $j^{\max }-1$ wavelet decomposition levels and by including the coarsest-scale scaling functions $\phi_{j^{\max }-1, k}^{(\cdot)}$. In (6), the (coarsest-scale) scaling functions correspond by convention to $\phi_{j_{\max }-1, k}^{()^{2}}=\psi_{j^{\max }, k}^{(\cdot)}$.

To apply the wavelet decomposition algorithm, we first need to approximate the reconstructed signal $\lambda$ in $\mathcal{V}_{0}$. Conventionally, the activity is represented as a time series of pixelated images. The choice of pixels in the spatial domain is related to how the projector $\mathrm{P}$ is (spatially) discretized. The choice of bins in the time domain may be imposed by the time resolution in the available data $y$ (list-mode format or a sequence of sinograms). We have chosen to keep this simple image model and we will denote it as

$$
\tilde{\lambda}\left(x_{1}, x_{2}, \tau\right)=\sum_{\boldsymbol{k}} \tilde{c}[\boldsymbol{k}] \zeta_{\boldsymbol{k}}\left(x_{1}, x_{2}, \tau\right)
$$

where the time pixel is given by $\zeta_{\boldsymbol{k}}\left(x_{1}, x_{2}, \tau\right)=\zeta^{(1)}\left(x_{1}-\right.$ $\left.k_{1}\right) \zeta^{(1)}\left(x_{2}-k_{2}\right) \zeta^{(2)}\left(\tau-k_{3}\right)$, with $\zeta^{(1)}$ and $\zeta^{(2)}$ boxcar functions and $\tilde{c}[k]$ are the corresponding coefficients. This choice may be imposed by the PET system and we will refer to the space spanned by the time pixels as the sampling space $\mathcal{V}_{\zeta}$. Note that this image model is still possible for sinogram data with nonuniform temporal bins by considering a uniform resampling of the data.

It is useful to think of $\tilde{c}[\boldsymbol{k}]$ as generalized samples of the reconstructed image $\lambda^{\text {recon }}\left(x_{1}, x_{2}, \tau\right) \in L_{2}$. The samples are obtained from an integration over the time pixel similar to (3). To avoid loss in spatial and temporal $(\Delta \tau)$ resolution the pixels are chosen small enough.

The coefficients $c_{0}[k]$ of the approximation in $\mathcal{V}_{0}$ can be obtained from the generalized samples $\tilde{c}[\boldsymbol{k}]$ by a simple prefiltering step, similar to the anti-aliasing filter in conventional sampling. In the case of separable basis functions, we can implement the filtering as separable 1-D filtering operations along the rows, columns, and time dimension of $\tilde{c}[\boldsymbol{k}]$. The 1-D prefilters $q[k]$ are designed using the principle of consistent sampling and are given by the convolutional inverse of the cross-correlation sequence $\left\langle\phi_{0}^{(\cdot)}(x-k), \zeta(x)\right\rangle$ [47].

When we include this prefiltering step explicitly in the update equation we get

$$
\tilde{\lambda}^{n+1}=\mathcal{N} \mathcal{Q}^{-1} \mathrm{~S}_{\mu}\left[\mathcal{Q}\left(\tilde{\lambda}^{n}+\mathrm{P}^{T}\left(y-\mathrm{P} \tilde{\lambda}^{n}\right)\right)\right]
$$

where $\mathcal{Q}$ denotes the prefiltering operation that maps an image from $\mathcal{V}_{\zeta}$ to $\mathcal{V}_{0}$ following the consistent sampling methodology, $\mathcal{Q}^{-1}$ represents the inverse operation. The projector on nonnegative functions $\mathcal{N}$ works in the pixelized space and therefore corresponds to setting pixels with negative values to zero.

\section{Filterbank Implementation}

The wavelet analysis and synthesis steps can be implemented by means of a filterbank [29]. Explicit expressions for designing the $E$-spline wavelet filters for given $\boldsymbol{\alpha}$ and $\gamma$ can be found in [21]. One important difference with $B$-spline wavelets is that the analysis and synthesis filters depend on the decomposition level $j$; this translates in selecting the appropriate precalculated filters at every decomposition level. We have used orthogonal wavelet transforms using IIR filters with exponential decay. The filtering operations were performed in the Fourier domain using the fast Fourier transform (FFT) algorithm. Since the considered wavelets are separable, the filterbank algorithm is applied successively to the rows, columns, and time dimension of the image. For the reconstruction of the simulated data, we used mirror boundary conditions in both space and time. The gated data were reconstructed using cyclic boundary conditions in the time domain.

\section{E. Shrinkage}

For the data reconstruction, we have set $\mu=$ const for all decomposition levels. The scaling-function coefficients were not penalized. The total number of temporal decomposition levels, $j_{3}^{\max }-1=J_{t}$, was kept constant for all experiments; i.e., $J_{t}=5$, instead mentioned otherwise. The total number of spatial decomposition levels was set to $j_{1}^{\max }-1=j_{2}^{\max }-1=$ $J_{s}$. Because the spatial resolution in the initial approximation space $\mathcal{V}_{0}^{(1)}$ is already relatively low (e.g., pixel size $3.13 \mathrm{~mm} \times$ $3.13 \mathrm{~mm}$ in the cardiac phantom), we have selected $J_{s}=1$. We also considered the case of pure temporal regularization, which can be seen as the special case $J_{s}=0$. To avoid the typical block artefacts when using the orthogonal DWT, we have applied wavelet cycle-spinning, as proposed in [18]. This method is easy to implement in the current setting; it aims at achieving some level of translation invariance by choosing a randomly-shifted DWT at each iteration of (21).

\section{EXPERIMENTAL EVALUATION}

\section{A. One-Dimensional Evaluation of the E-Spline Wavelets}

To illustrate the possibilities of the $E$-spline wavelets in dynamic PET reconstruction, we performed a 1-D denoising experiment on a left and right ventricular blood pool time activity curve as encountered in dynamic $\left[{ }^{13} \mathrm{~N}\right]-\mathrm{NH}_{3}$ PET [43]. The two time activity curves were given by exponential polynomials [43]

$$
\lambda^{\text {true }}(\tau)=\tau^{2} e^{-a \tau}+c s t
$$

with $a$ is 0.2 and 0.4 for the left ventricle (LV) and right ventricle (RV), respectively. Fig. 4 depicts scaled versions of the TACs. The poles and zero's of the system with the used curves as impulse response are given in Table II. The table also indicate the cutoff frequency. The cutoff frequency corresponds, in a first-order approximation, to the frequency where the slope of the amplitude characteristic changes.

The wavelet decomposition consisted of five levels and we included cycle spinning in the procedure. We used orthogonal $E$-spline wavelets with $\boldsymbol{\alpha}=\{0, \alpha, \alpha\}$ where $\alpha$ ranged from 0 to -1 in steps of -0.05 . Note that we have used here a multiplicity of 2 instead of 3 for the pole $\alpha$ in order to illustrate the robustness of the parameter selection.

Additionally we have considered modified versions of the right ventricular input curve. First we reduced the multiplicity of the pole -0.4 , which corresponds to replacing $\tau^{2}$ with $\tau$ in 
TABLE II

POLES AND ZEROS AND THE CORRESPONDING CUT-OFF FREQUENCY $\omega_{0}$ OF THE RIGHT AND LEFT VENTRICULAR RESPONSE. THE IDEALIZED $\boldsymbol{\alpha}$ AND $\boldsymbol{\gamma}$ ARE COMPOSED OF THE ROOTS WITH MULTIPLICITY $m_{l}$ CORRESPONDING TO THE POLES AND ZEROS, RESPECTIVELY

\begin{tabular}{|c|c|c|c|c|}
\hline Region & $\omega_{0}$ & $\mathrm{p} / \mathrm{z}$ & $m_{l}$ & root \\
\hline \hline \multirow{4}{*}{ RV } & 0 & pole & 1 & 0 \\
\cline { 2 - 5 } & 0.01 & zero & 1 & -0.01 \\
\cline { 2 - 5 } & 0.4 & pole & 3 & -0.4 \\
\cline { 2 - 5 } & 2.6 & zero & 1 & $-0.59+2.34 i$ \\
\cline { 2 - 5 } & 2.6 & zero & 1 & $-0.59-2.34 i$ \\
\hline \hline \multirow{4}{*}{ LV } & 0 & pole & 1 & 0 \\
\cline { 2 - 5 } & 0.006 & zero & 1 & -0.006 \\
\cline { 2 - 5 } & 0.2 & pole & 3 & -0.2 \\
\cline { 2 - 5 } & 1.4 & zero & 1 & $-0.30+1.10 i$ \\
\cline { 2 - 5 } & 1.4 & zero & 1 & $-0.30-1.10 i$ \\
\hline
\end{tabular}

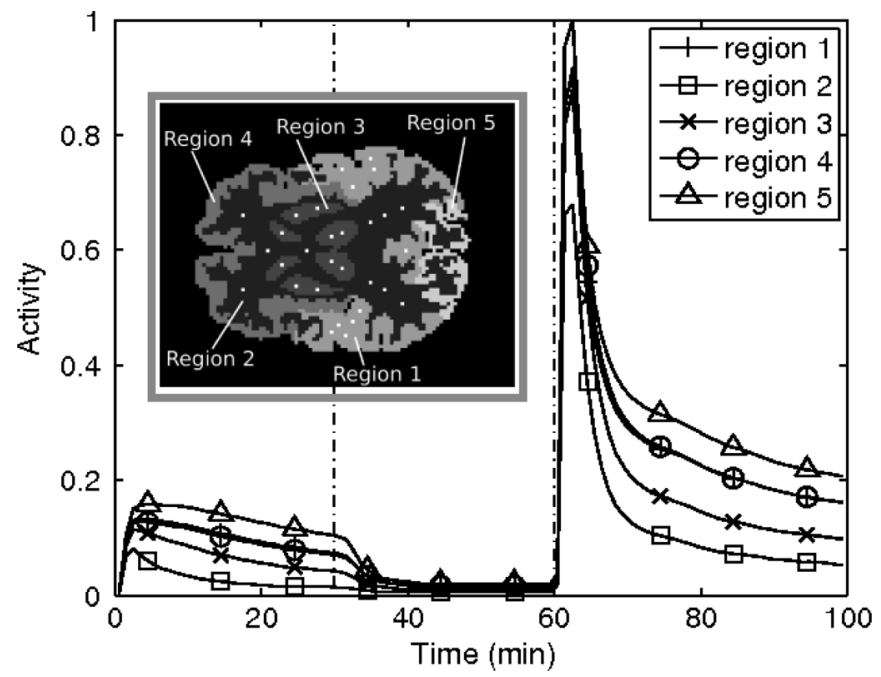

Fig. 6. Brain $\left[{ }^{11} \mathrm{C}\right]-$ FMZ imaging simulation experiment. TACs for the different regions. Vertical lines at 30 and $60 \mathrm{~min}$ indicate the displacement injection and the coinjection times, respectively. And slice of the Zubal phantom $(22 \mathrm{~cm}$ $\times 18 \mathrm{~cm}$ ) with the positions of the pixels used for the analysis.

(26) $\left(\operatorname{tac}_{1}\right)$. We also included a curve $\left(\operatorname{tac}_{2}\right)$ without a recirculation term $(c s t=0)$. For the latter, we have also considered the parameter vector $\boldsymbol{\alpha}=\{\alpha, \alpha, \alpha\}$.

The 1-D denoising experiment was also performed for a TAC derived from a $\left.{ }^{11} \mathrm{C}\right]$ - flumazenil (FMZ) brain PET study [48]. The TAC corresponds to region 1 in Fig. 6. We used orthogonal $E$-spline wavelets with $\boldsymbol{\alpha}=\{0, \alpha, \alpha\}$ where $\alpha$ ranged from 0 to -2 in steps of -0.1 .

The TACs were divided into 100 time bins and 1000 Poisson realisations were generated. The curves were scaled to achieve a data SNR of around 10 and $20 \mathrm{~dB}$. We evaluated the SNR of the curves reconstructed from the $N$ largest wavelet and scaling coefficients $\left(\ell_{0}\right.$-norm). The SNR was calculated from the samples at $\tau_{k}=\{(2 k+1) / 2\}_{k=0}^{99}$ as

$$
\mathrm{SNR}=10 \log \frac{\sum_{k} \lambda^{\text {true }}\left(\tau_{k}\right)^{2}}{\sum_{k}\left(\lambda^{\operatorname{true}}\left(\tau_{k}\right)-\hat{\lambda}\left(\tau_{k}\right)\right)^{2}}
$$

where $\hat{\lambda}\left(\tau_{k}\right)$ are the samples of the denoised TAC.
To test the robustness, we have calculated the fraction of tested exponential parameter vectors $\boldsymbol{\alpha}$ that outperformed the $B$-spline wavelets with at least $5 \%$ in terms of reconstructed SNR.

\section{B. Tomographic Simulation Studies}

We considered two $2+1-\mathrm{D}$ (spatial + temporal) tomographic simulations, a $\left[{ }^{13} \mathrm{~N}\right]-\mathrm{NH}_{3}$ cardiac and $\left[{ }^{11} \mathrm{C}\right]$-flumazenil (FMZ) brain imaging experiment, respectively. The cardiac phantom consisted of 100 time frames of $100 \times 80$ pixels $(3.13 \mathrm{~mm} \times$ $3.13 \mathrm{~mm} \times 1 \mathrm{~s})$. The phantom was a slice of the NCAT phantom containing the myocardium [49]. The phantom consisted of four regions and four TACs were simulated for the left and right ventricle, the myocardium and the background, respectively. The time curves in the ventricles were given by the exponential polynomials, as in Section V-A. The time curves of the myocardium were derived from the left ventricular input function using a two compartmental model [43]. The phantom and the time curves are illustrated in Fig. 4.

The brain phantom consisted of 100 time frames of $100 \times$ 80 pixels $(2.2 \mathrm{~mm} \times 2.2 \mathrm{~mm} \times 1 \mathrm{~min})$. The phantom was a mid brain slice of the Zubal brain phantom [50]. Five different TACs were simulated as illustrated in Fig. 6. The kinetics of the data are based on a multi-injection protocol using $\left[{ }^{11} \mathrm{C}\right]-\mathrm{FMZ}$ for quantification of benzodiazepine receptors [51]. The TACs were synthesized from a measured input curve [48] and the 5 parameter compartmental ligand-receptor model [51]. The different responses were obtained using typical parameter values for different regions. The five regions correspond to the temporal cortex, pons, cerebellum, frontal, and occipital cortex for region $1-5$, respectively. At times $\tau=0 \mathrm{~min}$ and $\tau=30 \mathrm{~min}$ labelled and unlabelled (displacement injection) ligand is injected, respectively. At $\tau=60 \mathrm{~min}$ both labelled and unlabelled ligand are injected (coinjection), as illustrated in Fig. 6. The sinogram data were generated using the decay corrected TACs.

The simulated PET system was set up as a geometric approximation of a commercial scanner consisting of 616 detector crystals in a ring (radius $43 \mathrm{~cm}$ ) [52]. Photons were emitted back-to-back according to an inhomogeneous Poisson process but no absorption and scatter effects were simulated.

We considered different $\boldsymbol{\alpha}$ 's for each experiment: 1) the $B$-spline wavelets using $\boldsymbol{\alpha}=\{0,0,0\}$, corresponding to cubic Battle-Lemarié wavelets and (2) $E$-spline wavelets of the form $\boldsymbol{\alpha}=\{0, \alpha, \alpha\}$ with $\alpha=-0.2, \alpha=-0.4$, and $\alpha=-0.6$ and with $\alpha=-0.5, \alpha=-1$, and $\alpha=-1.5$ for the cardiac and brain imaging reconstructions, respectively.

We simulated 10 noise realizations of both phantoms. Each realization consisted of about 800000 and 1400000 detected pairs for the cardiac and brain study, respectively. The reconstructions were obtained after 100 iterations of (21) with $\lambda^{0}$ equal to zero. For each experiment, the reconstructed SNR following (27) with $\hat{\lambda}\left(x_{1}, x_{2}, \tau_{k}\right)$, the samples of the reconstructed TAC at location $\left(x_{1}, x_{2}\right)$ was evaluated. There were 30 pixel locations, 10 per representative region (left and right ventricle and left myocardium and region 1-3 for the two experiments, respectively). The pixel locations are illustrated in Fig. 4 and 6. Thus, for the all regions we had 10 (ROIs) $\times 10$ (realizations) reconstructed TACs. 

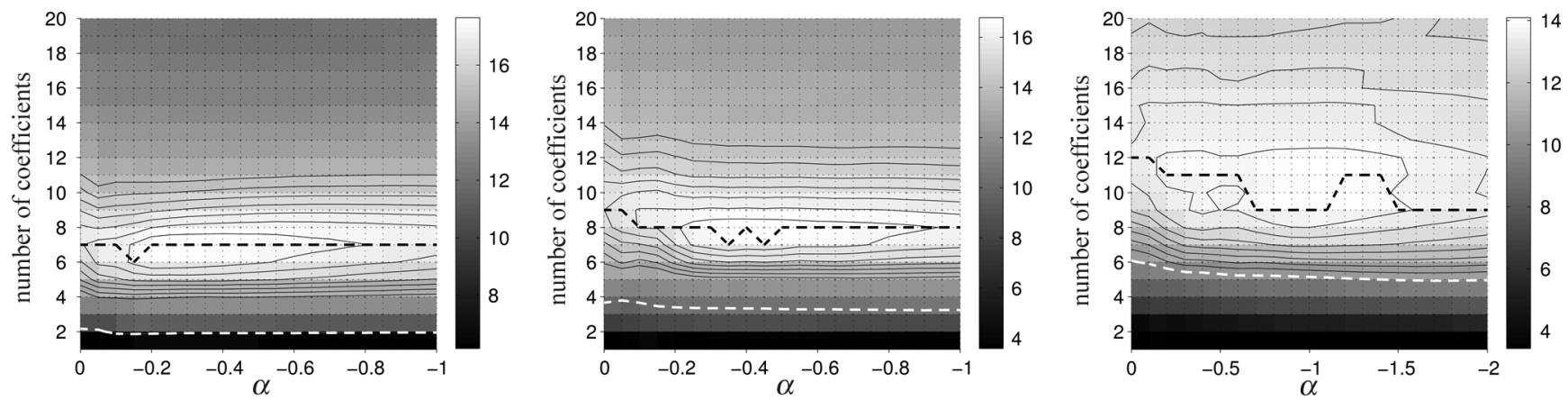

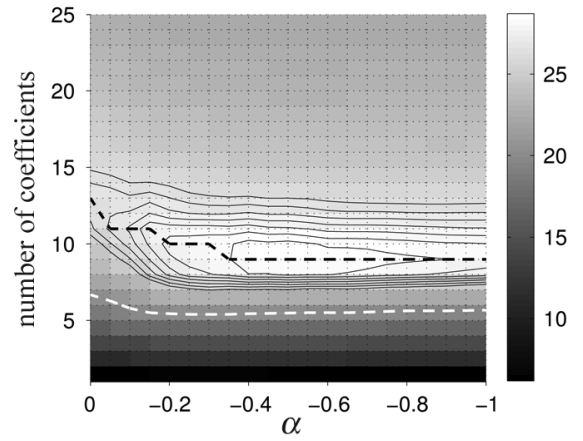

(a)

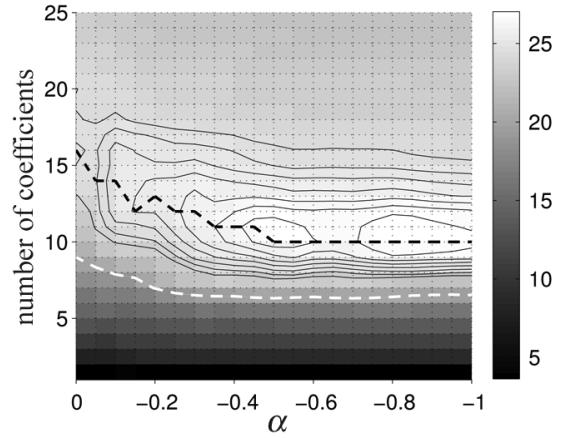

(b)

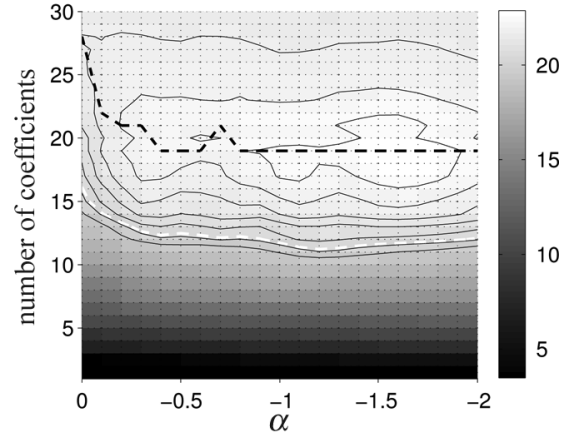

(c)

Fig. 7. One-dimensional denoising results for the right (a) and left (b) ventricle of the heart phantom and for region 1 (c) of the brain phantom. SNR as a function of the number of nonzero coefficients and $\alpha=\{0, \alpha, \alpha\}$. Top: input SNR around $10 \mathrm{~dB}$ and bottom: input SNR around $20 \mathrm{~dB}$. Seven contour lines are drawn with intervals of $0.5 \mathrm{~dB}$. Input noise level is illustrated by the white dashed line. The number of non-zero coefficients achieving the highest SNR for different $\alpha$ parameters is illustrated by the black dashed line. $E$-spline wavelet denoising can attain a higher reconstructed SNR using less coefficients.

TABLE III

ONE-DIMENSIONAL DENOISING RESULTS. OPTIMAL $\alpha$ AND THE RESPECTIVE GAINS IN SNR AND NUMBER OF NONZERO COEFFICIENTS COMPARED TO THE RESULTS USING $B$-SPLINE WAVELETS $(\alpha=0)$

\begin{tabular}{|c|c|c|c|c|c|c|}
\hline Region & $\begin{array}{c}\text { input } \\
\mathrm{dB}\end{array}$ & $\boldsymbol{\alpha}$ & $\begin{array}{c}\text { optimal } \\
\alpha\end{array}$ & $\begin{array}{c}\text { gain } \\
\mathrm{dB}\end{array}$ & $\begin{array}{c}\text { gain } \\
\text { coeff }\end{array}$ & $\begin{array}{c}\Delta \alpha[\%] \\
\mathrm{SNR}+5 \%\end{array}$ \\
\hline \hline \multirow{2}{*}{$\mathrm{LV}$} & 10 & $\{0, \alpha, \alpha\}$ & -0.25 & +1.1 & 0 & 35 \\
& 20 & $\{0, \alpha, \alpha\}$ & -0.50 & +2.7 & 4 & 90 \\
\hline \multirow{2}{*}{$\mathrm{RV}$} & 10 & $\{0, \alpha, \alpha\}$ & -0.35 & +1.5 & 2 & 85 \\
& 20 & $\{0, \alpha, \alpha\}$ & -0.85 & +2.9 & 6 & 80 \\
\hline \multirow{2}{*}{$\operatorname{tac}_{1}$} & 10 & $\{0, \alpha, \alpha\}$ & -0.45 & +1.9 & 2 & 80 \\
& 20 & $\{0, \alpha, \alpha\}$ & -0.55 & +3.5 & 8 & 75 \\
\hline \multirow{3}{*}{$\operatorname{tac}_{2}$} & 10 & $\{0, \alpha, \alpha\}$ & -0.35 & +1.7 & 1 & 100 \\
& 20 & $\{0, \alpha, \alpha\}$ & -0.45 & +2.3 & 5 & 80 \\
\cline { 2 - 7 } & 10 & $\{\alpha, \alpha, \alpha\}$ & -0.40 & +2.9 & 2 & 75 \\
& 20 & $\{\alpha, \alpha, \alpha\}$ & -0.50 & +3.4 & 7 & 60 \\
\hline \multirow{2}{*}{$\operatorname{Region}^{*} 1$} & 10 & $\{0, \alpha, \alpha\}$ & -1 & +0.8 & 3 & 25 \\
& 20 & $\{0, \alpha, \alpha\}$ & -1.6 & +1.5 & 9 & 45 \\
\hline
\end{tabular}

\section{Clinical Data}

We have reconstructed two different clinical data sets. One dynamic $\left[{ }^{18} \mathrm{~F}\right]$-FDG study of the liver and one gated cardiac $\left[{ }^{18} \mathrm{~F}\right]$-FDG study.

The 45 min dynamic liver study was acquired at the Ghent University Hospital on a Philips Allegro PET/CT scanner [52]. The data were acquired in list-mode format and were then binned into 90 temporal frames of $30 \mathrm{~s}$. To reduce the computational cost, we used single slice rebinning (SSRB) to create a stack of 3-D (2-D spatial + 1-D temporal) transaxial sinograms. The SSRB [53] maps the oblique sinogram data into equivalent transaxial sinograms. We reconstructed every transaxial plane separately. The data were precorrected for randoms using a smoothed version of the delayed events; after randoms subtraction $530 \mathrm{M}$ counts were left. Attenuation precorrection was performed using the map available from the scanner and generated from the CT image. We did not consider scatter correction. The data were reconstructed in 90 temporal frames of 30 slices ( $6 \mathrm{~mm}$ thick). The slices were $144 \times 144$ $(4 \mathrm{~mm} \times 4 \mathrm{~mm})$.

For this study, we have selected $\boldsymbol{\alpha}=\{0,-0.6,-0.6\}$. We have used mirror boundary conditions in the temporal and spatial domain. The wavelet decomposition was made with three decomposition levels $\left(J_{s}=J_{t}=3\right)$.

We also considered a more conventional reconstruction we have binned the data for the first 34 min into 17 nonuniform time frames. The shortest time frames were used at the beginning of the scan. There were six frames of $30 \mathrm{~s}$, three frames of $1 \mathrm{~min}$, two frames of $2 \mathrm{~min}$, and six frames of $4 \mathrm{~min}$. The nonuniformly binned data were preprocessed in the same way as the uniformly binned data. The 17 time frames were reconstructed independently without any spatial regularization. To compare these reconstructions to the reconstructions using the $\ell 1$ spatio-temporal wavelet regularization, we have postsmoothed the 17 individual time frames using a 2-D Gaussian filter (smoothing in the spatial domain). The FWHM of the Gaussian filter was chosen so that the reconstructed images had approximately the same spatial resolution at the injection site.

To further illustrate the range of applicability of our method, we have performed reconstructions of a gated cardiac PET study 

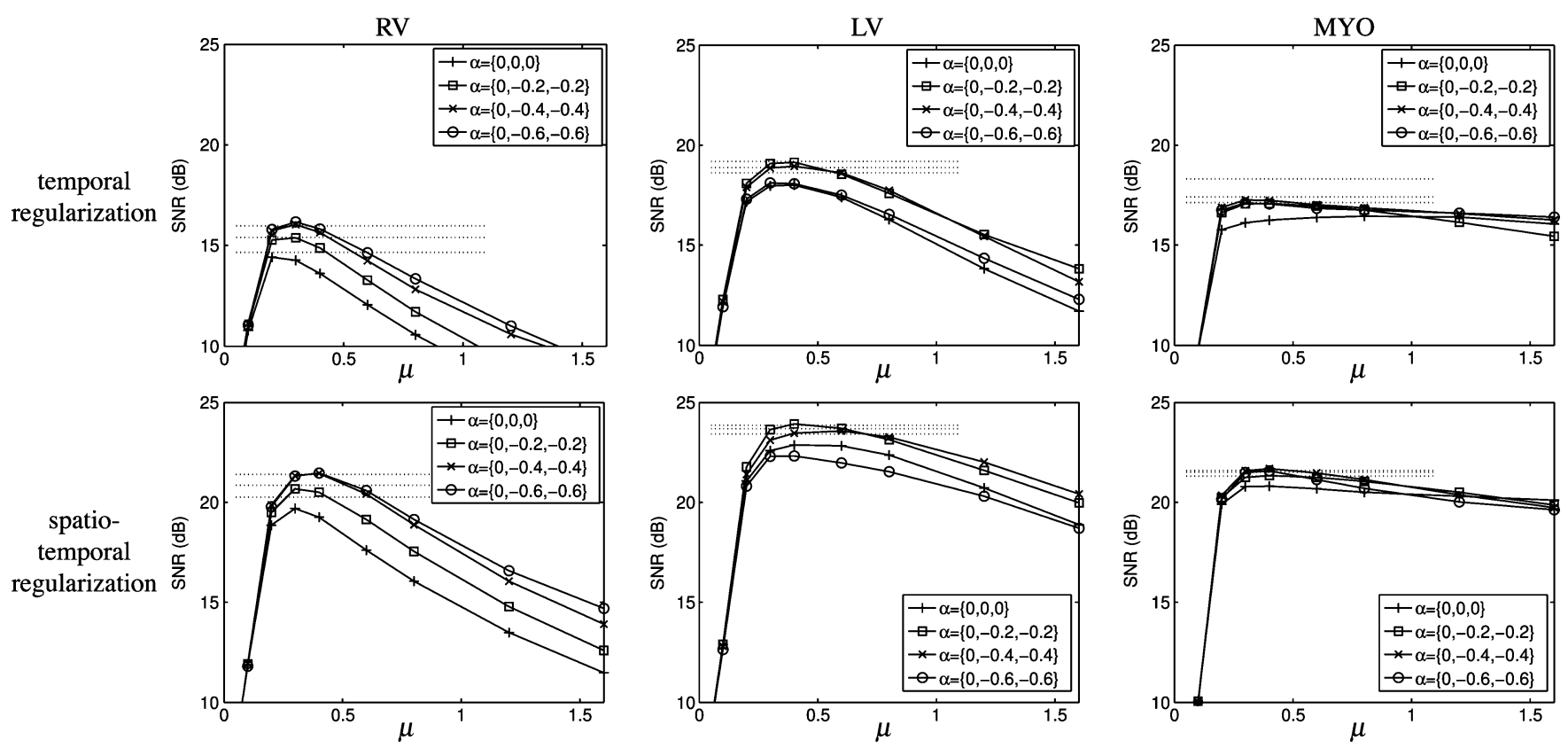

Fig. 8. Tomographic reconstruction results of the cardiac imaging simulation. SNR as a function of $\mu$ for the three regions and for a different number of spatial decomposition levels. SNR obtained using the $B$-spline wavelets $(\boldsymbol{\alpha}=\{0,0,0\})$ compared to the SNR obtained using $E$-spline wavelets $(\boldsymbol{\alpha}=\{0,-0.2,-0.2\}$, $\boldsymbol{\alpha}=\{0,-0.4,-0.4\}, \boldsymbol{\alpha}=\{0,-0.6,-0.6\}$ ). Dotted lines denote the maximal attainable SNR using $\boldsymbol{\alpha}=\{0,0,0\}$ for data sets with $20 \%, 40 \%$, and $60 \%$ more counts, respectively. Completely unregularized solutions corresponding to $\mu=0$ attained SNRs of less then $10 \mathrm{~dB}$. It can be observed that appropriately chosen $E$-spline wavelets consistently outperform $B$-spline wavelets.

acquired at the Geneva University Hospital. The data were acquired on a Siemens Biograph PET/CT scanner [54] and the cardiac cycle was divided into 20 gates. We have used SSRB to reduce the computational cost. The original sinogram data contained $80 \mathrm{M}$ counts. After SSRB with a maximal ring difference of 16 this was reduced to $19 \mathrm{M}$ counts. The data were precorrected for randoms and for attenuation using an attenuation map derived from the CT image [55]. We did not consider scatter correction. We have reconstructed 40 slices ( $4 \mathrm{~mm}$ thick). The slices were $184 \times 184(2.5 \mathrm{~mm} \times 2.5 \mathrm{~mm})$.

In this study, the dynamics of the data are caused by the motion of the heart rather than by the activity exchange between compartments. The dynamics are rather slow which led us to select $\boldsymbol{\alpha}=\{0,0,0\}$; i.e., $B$-spline wavelets, which are a special case of our framework. This example allows us to illustrate the effect of temporal regularization. We have used periodic boundary conditions in the temporal domain to reflect the periodicity of the heart phase. As before, mirror boundary conditions were applied in the spatial domain. The wavelet decomposition was made with three decomposition levels $\left(J_{s}=J_{t}=3\right)$.

\section{RESULTS}

\section{A. One-Dimensional Evaluation of the E-Spline Wavelets}

The results for the 1-D study are shown in Fig. 7 for the left and right ventricle and the region 1, respectively. $B$-spline wavelets corresponds to $\alpha=0$.

For high noise levels (close to $10 \mathrm{~dB}$ ), the best results are obtained with a low number of coefficients (less than 12), leading to a very sparse solution. The best performance is obtained for $\alpha=-0.25, \alpha=-0.35$, and $\alpha=-1$ and is about 1.1, 1.5, and
TABLE IV

IMPROVEMENT IN RECONSTRUCTED SNR USING $E$-SPLINE WAVELETS COMPARED TO $B$-SPLINE WAVELETS $(\boldsymbol{\alpha}=\{0,0,0\})$ FOR THE CARDIAC IMAGING $(\boldsymbol{\alpha}=\{0,-0.4,-0.4\})$ AND BRAIN IMAGING SIMULATIONS $(\boldsymbol{\alpha}=\{0,-1.5,-1.5\})$. High NoISE Results (Two TIMES Less CounTS) ARE DENOTED BY *. TUNing PARAMETER IS $\mu=0.3$ AND $\mu=1.2$, RESPECTIVELY

\begin{tabular}{|c|c|c||c|c|}
\hline \multirow{2}{*}{ Region } & \multicolumn{2}{|c||}{$\mu=0.3$} & \multicolumn{2}{c|}{$\mu=1.2$} \\
& $\begin{array}{c}\text { temporal } \\
\text { regularization }\end{array}$ & $\begin{array}{c}\text { spatio-temporal } \\
\text { regularization }\end{array}$ & $\begin{array}{c}\text { temporal } \\
\text { regularization }\end{array}$ & $\begin{array}{c}\text { spatio-temporal } \\
\text { regularization }\end{array}$ \\
\hline \hline $\mathrm{RV}$ & $+1.76 \mathrm{~dB}$ & $+1.63 \mathrm{~dB}$ & $+2.45 \mathrm{~dB}$ & $+2.57 \mathrm{~dB}$ \\
\hline $\mathrm{LV}$ & $+0.88 \mathrm{~dB}$ & $+0.53 \mathrm{~dB}$ & $+1.60 \mathrm{~dB}$ & $+1.30 \mathrm{~dB}$ \\
\hline MYO & $+1.13 \mathrm{~dB}$ & $+0.80 \mathrm{~dB}$ & $+0.18 \mathrm{~dB}$ & $+0.10 \mathrm{~dB}$ \\
\hline \hline $\mathrm{RV}^{*}$ & $+1.62 \mathrm{~dB}$ & $+2.21 \mathrm{~dB}$ & $+2.17 \mathrm{~dB}$ & $+2.70 \mathrm{~dB}$ \\
\hline $\mathrm{LV}^{*}$ & $+1.09 \mathrm{~dB}$ & $+0.96 \mathrm{~dB}$ & $+0.93 \mathrm{~dB}$ & $+1.59 \mathrm{~dB}$ \\
\hline $\mathrm{MYO}^{*}$ & $+1.36 \mathrm{~dB}$ & $+0.85 \mathrm{~dB}$ & $+1.22 \mathrm{~dB}$ & $-0.51 \mathrm{~dB}$ \\
\hline \hline R 1 & $+0.52 \mathrm{~dB}$ & $+0.24 \mathrm{~dB}$ & $+0.93 \mathrm{~dB}$ & $+0.53 \mathrm{~dB}$ \\
\hline $\mathrm{R} 2$ & $+0.77 \mathrm{~dB}$ & $+0.60 \mathrm{~dB}$ & $+1.49 \mathrm{~dB}$ & $+1.11 \mathrm{~dB}$ \\
\hline $\mathrm{R} 3$ & $+0.69 \mathrm{~dB}$ & $+0.32 \mathrm{~dB}$ & $+1.16 \mathrm{~dB}$ & $+0.8 \mathrm{~dB}$ \\
\hline
\end{tabular}

$0.8 \mathrm{~dB}$ better than the results for the $B$-spline wavelets for the left and right ventricle and the region 1 , respectively.

A higher number of coefficients can be used for the lower noise level (around $20 \mathrm{~dB}$ ). For $\alpha<0$, the best results are obtained for a smaller number of coefficients as compared to the $B$-spline wavelets. For the left ventricle, the best results are obtained using $\alpha=-0.5$ with nine wavelet coefficients only whereas the best results for the $B$-spline wavelets are found when using 13 coefficients. This illustrates that a sparser representation is possible when using appropriate $E$-spline wavelets. Moreover, the best performance for $\alpha=-0.5$ is about $2.7 \mathrm{~dB}$ better than for the best performance using $\alpha=0$. Similar results 

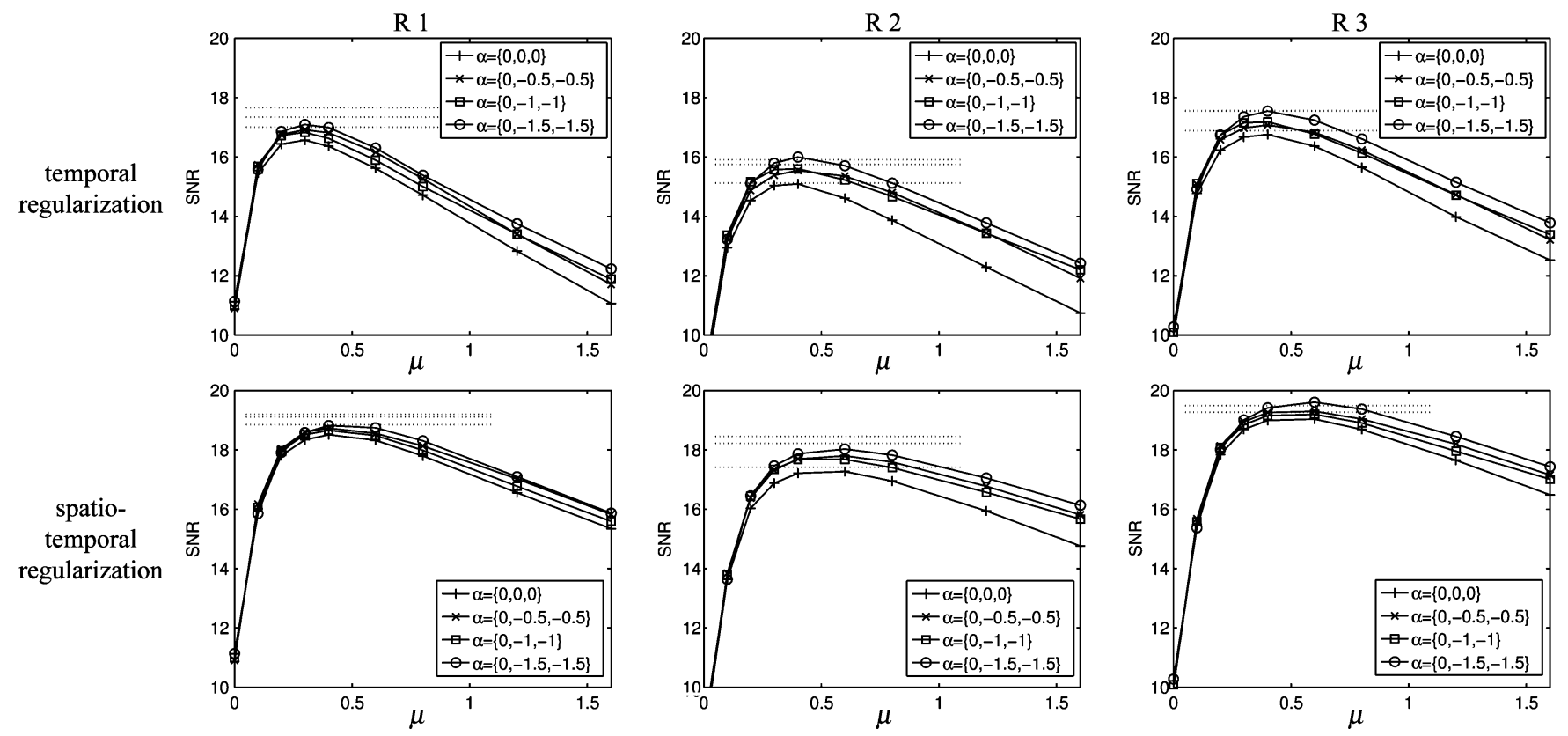

Fig. 9. Tomographic reconstruction results of the brain imaging simulation. SNR as a function of $\mu$ for the three regions and for a different number of spatial decomposition levels. SNR obtained using the $B$-spline wavelets $(\boldsymbol{\alpha}=\{0,0,0\})$ compared to the SNR obtained using $E$-spline wavelets $(\boldsymbol{\alpha}=\{0,-0.5,-0.5\}$, $\alpha=\{0,-1,-1\}, \alpha=\{0,-1.5,-1.5\}$ ). Dotted lines denote the maximal attainable SNR using $\alpha=\{0,0,0\}$ for data sets with $20 \%, 40 \%$, and $60 \%$ more counts, respectively. It can be observed that $E$-splines consistently outperform $B$-spline wavelets.

are obtained for the right ventricle. The maximal SNR gain of the $E$-splines in this case was about $2.9 \mathrm{~dB}$ for $\alpha=-0.85$ and required six coefficients less compared to the $B$-spline results. The results for the modified curves and region 1 are summarized in Table III.

Note that for the more complex TAC of region 1 there are more coefficients required compared to the TACs of the LV and RV.

Finally, from Fig. 7 and Table III, it can be seen that there is a broad range of $\alpha$ 's that yields reasonably sparse representations and that perform at least $5 \%$ better than the $B$-splines in terms of reconstructed SNR.

\section{B. Tomographic Simulation Study}

The results for the tomographic reconstruction of the $\left[{ }^{13} \mathrm{~N}\right]-\mathrm{NH}_{3}$ simulation data are shown in Fig. 8. Again, the highest reconstructed SNR is obtained when using the appropriate $E$-spline wavelets. The best results for the right ventricle are found for $\alpha=-0.6$ and are only slightly better than the results for $\alpha=-0.4$. For $\alpha=-0.2$, the results are in between the results for $\alpha=-0.6$ and the $B$-spline wavelets $(\alpha=0)$. The best results for the left ventricle are found for $\alpha=-0.2$ and are only slightly better than the results for $\alpha=-0.4$. The results for $\alpha=-0.6$ are slightly worse than the results for the $B$-spline wavelets $(\alpha=0)$. For the myocardium all the different $E$-spline wavelets perform equally well. The results for the three different curves reconstructed with the three different exponential spline parameters illustrate the robustness of the parameter choice.

A good compromise for the tomographic reconstruction is $\boldsymbol{\alpha}=\{0,-0.4,-0.4\}$. With this particular parameter vector, the reconstructed SNR for the different regions is improved as summarized in Table IV. We have also calculated the gains for a high noise study containing two times less detected counts $(\approx 400000)$ than the original study. Fig. 8 also illustrates the highest attainable SNR when using $\boldsymbol{\alpha}=\{0,0,0\}$ for the reconstruction of data sets with $20 \%, 40 \%$, and $60 \%$ more counts, respectively. Replacing the $B$-spline wavelets by the well tuned $E$-spline wavelets correspond to a $20 \%, 40 \%$, and $60 \%$ increase in counts for the myocardium, left ventricle and right ventricle, respectively.

Although the original TACs used for the $\left[{ }^{11} \mathrm{C}\right]-\mathrm{FMZ}$ simulation data, which are obtained from a linear interpolation of measured data, are not exactly exponential polynomials, we found that all tested $E$-spline wavelets $(\alpha=-0.5,-1,-1.5)$ outperformed the $B$-spline wavelets in terms of reconstructed SNR as illustrated in Fig. 9. This was true for TACs of the three different regions, illustrating the robustness of the parameter choice. The improvements for reconstructions using the two types of wavelets are summarized in Table IV. The improvements obtained by the $E$-spline wavelets is comparable to a $20 \%$ increase of detected counts as indicated by the dotted lines in Fig. 9.

In Fig. 10 temporal profiles for a pixel located in the left ventricle are illustrated. For these curves, no spatial regularization was applied. A regularized $(\mu=0.2)$ and unregularized $(\mu=0)$ profile of a single pixel are depicted along with a mean regularized profile. The mean profile was obtained as the mean over the 10 pixel ROIs (Fig. 4) and over the 10 different noise realizations.

Reconstructed slices using different spatial levels of decompositions are compared to the nonregularized solution in Figs. 11 and 12 . 


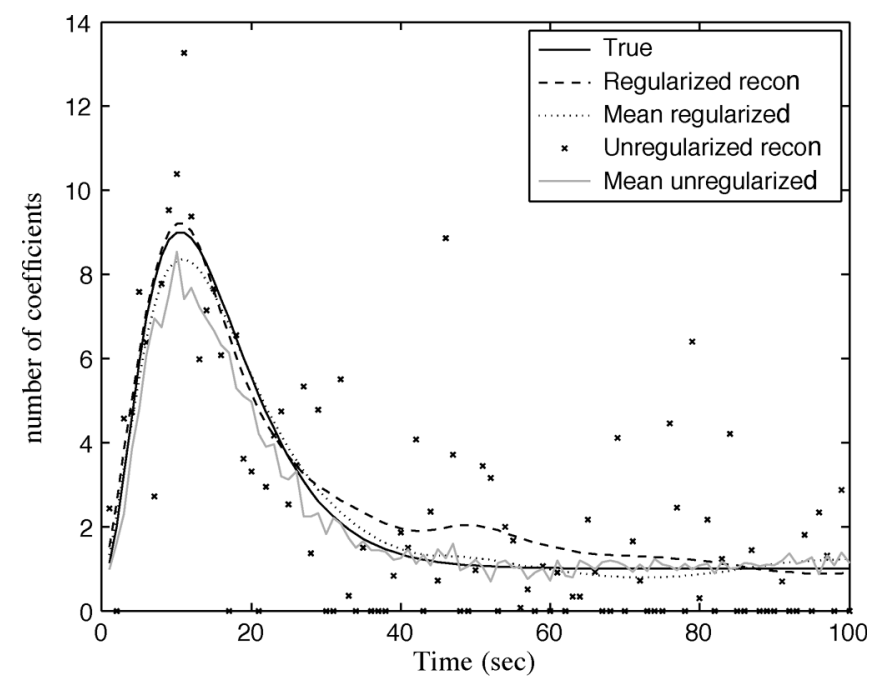

(a)

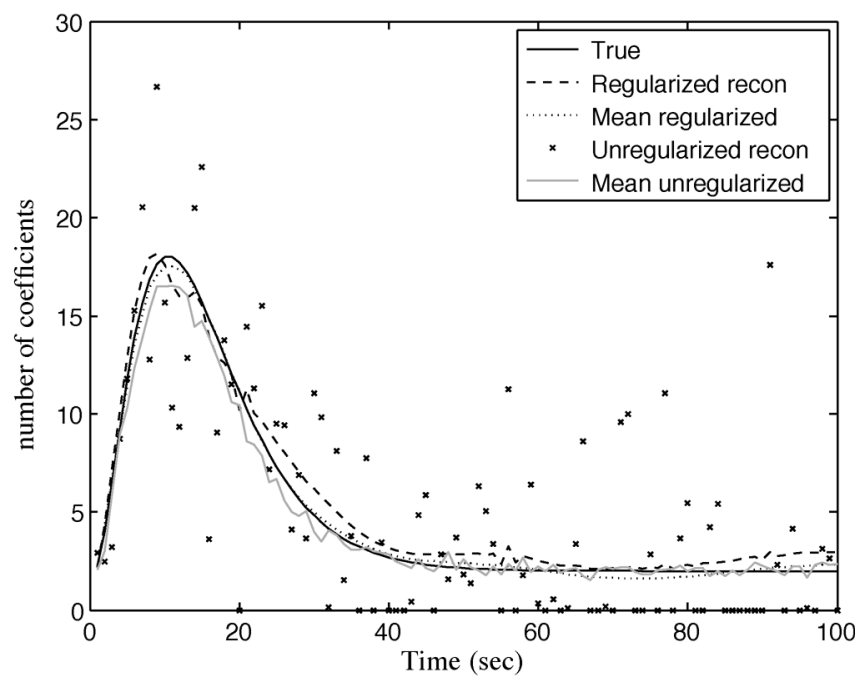

(b)

Fig. 10. Temporal profile for a pixel located in the left ventricle. Reconstructions are for $\boldsymbol{\alpha}=\{0,0,0\}$. Regularized reconstruction is for $\mu=0.2$ with only temporal regularization. Mean regularized reconstruction is the mean over all 10 pixel ROIs (Fig. 4) and over the 10 realizations. (a) Time activity curves for the low counts study. (b) Time activity curves for the high counts study.

\section{Clinical Data}

Reconstructed transverse slices, 45,285 , and 1185 s postinjection, of the dynamic liver data are illustrated in Fig. 13. The slice position is chosen so that the slice contains the liver, a kidney and the injection site. A 30-min-long temporal slice illustrated in Fig. 13. The reconstructions are obtained after 100 iterations and using tuning parameters $\mu=10, \mu=15$, and $\mu=20$, respectively. As a comparison, a reconstruction using nonuniform temporal frames without any additional temporal regularization is shown. The individual reconstructed nonuniform frames were postsmoothed with a Gaussian filter. The FWHM of the Gaussian filter was chosen so that all reconstructed images had approximately the same spatial resolution at the injection site. Finally, the nonuniform frames were linearly interpolated to correspond to uniform time frames of $30 \mathrm{~s}$.

From Fig. 13, the different noise levels and spatial resolution of the different reconstruction can be appreciated. The recon-

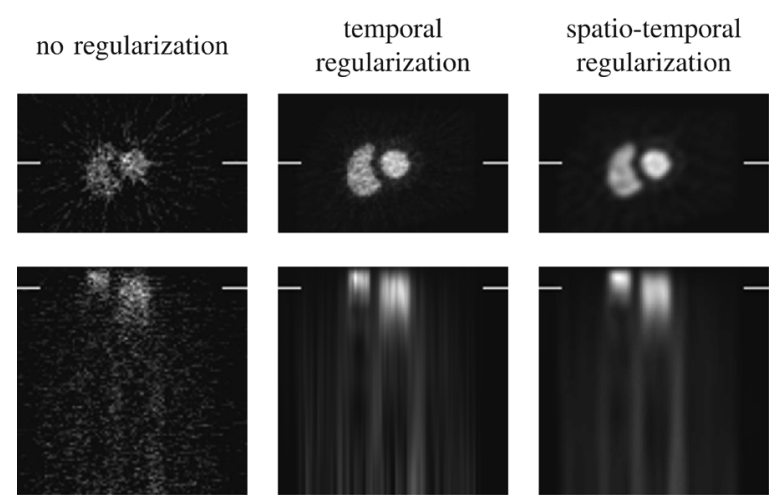

Fig. 11. Reconstructed slices using different number of spatial decomposition levels using $E$-spline wavelets with $\alpha=\{0,-0.4,-0.4\}$. Upper and lower rows are spatial and temporal slices, respectively. The time and space locations are indicated by the white bars.

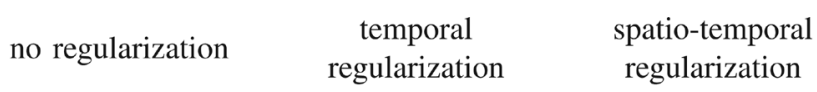
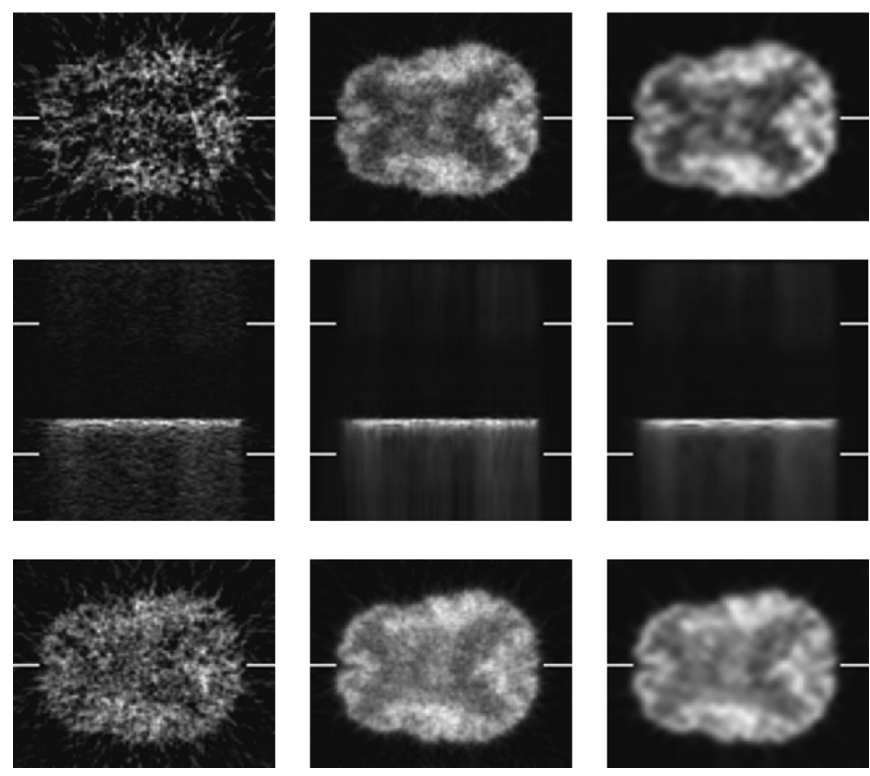

Fig. 12. Reconstructed slices using different number of spatial decomposition levels and tuning parameter $\mu$ using $E$-spline wavelets with $\alpha=\{0,-1,-1\}$. Middle row are temporal slices. Time and space locations are indicated by the white bars. Upper and lower spatial slices correspond with the upper (early time) and lower (late time) bars in the temporal slice, respectively. Results in the third column give a good compromise between spatial and temporal regularization.

structions using the $E$-spline wavelets have lower noise. While for $\mu=20$ there is a low spatial resolution, the reconstruction for $\mu=15$ and $\mu=10$ have resolution characteristics comparable to the postsmoothed reconstruction.

Temporal profiles of single pixels are illustrated in Fig. 14. The pixels are located in the injection site, the aorta, the liver, and kidney. Note that for display we have scaled down the TAC in the injection site by a factor 0.5 . The profiles for reconstructions using $\mu=10$ and $\mu=15$ are compared to the postsmoothed reconstructions using the nonuniform time frames. Again the different noise levels can be appreciated.

Transaxial and transverse slices of the reconstructions of the gated data are illustrated in Figs. 15 and 16. The reconstructions are at $0 \%$ and $40 \%$ of the cardiac cycle and are obtained 

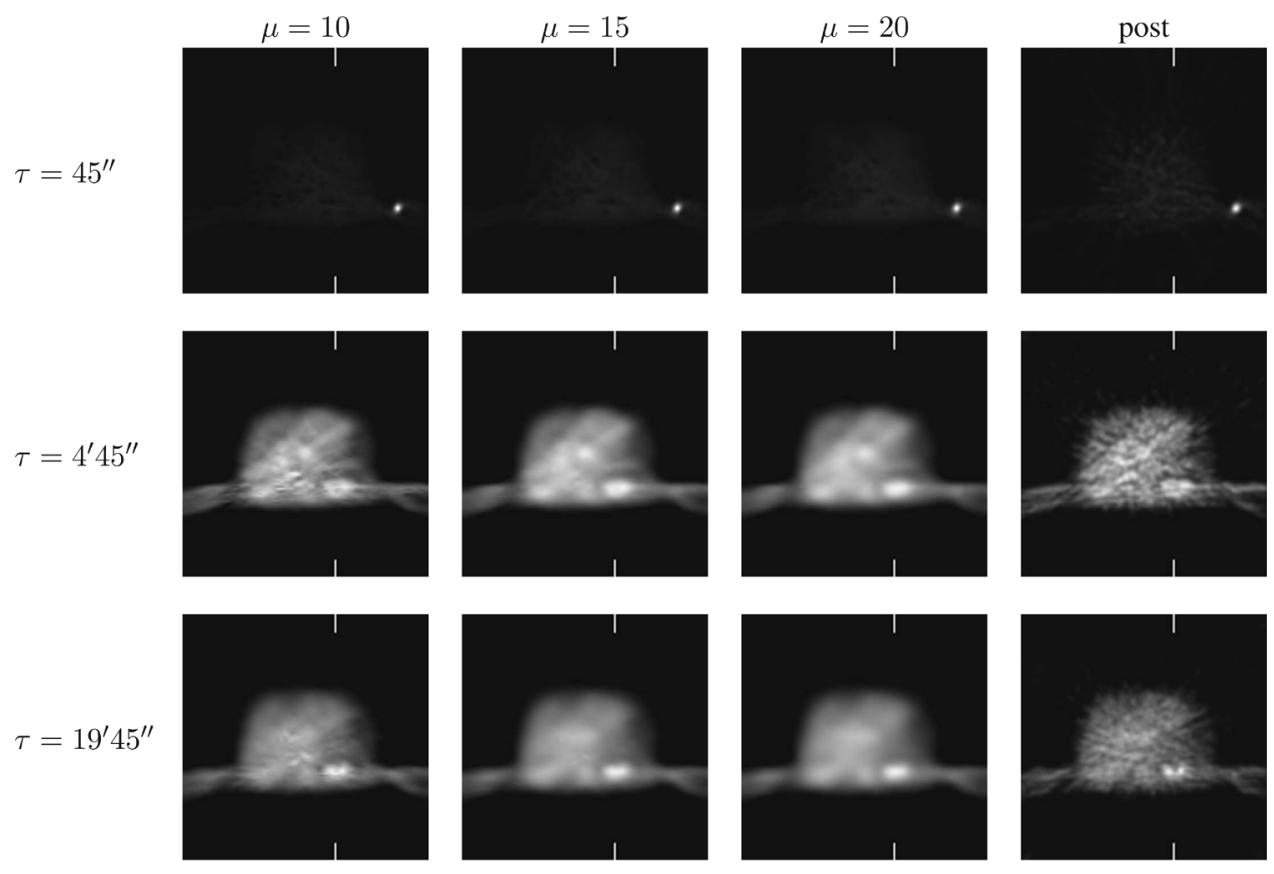

$x=72 \mathrm{~mm}$
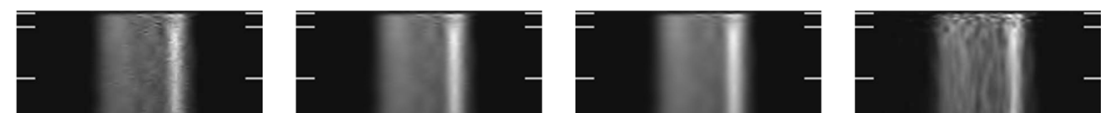

Fig. 13. Reconstructed slices of the liver data. Top three rows: transverse slices 45, 285, 1185 s postinjection (from top to bottom). Bottom row: temporal slice. Time locations of the transverse slices are indicated by the horizontal bars. Location of the temporal slices is illustrated by the white vertical bars. The reconstructions are obtained after 100 iterations and using tuning parameters $\mu=10, \mu=15$, and $\mu=20$, respectively. Postsmoothed reconstruction of the nonuniform time frame data is illustrated in the right most column (post).

after 200 iterations using tuning parameters $\mu=0.1$ and $\mu=$ 0.3 for Figs. 15 and 16, respectively. It is apparent that the increased tuning parameter $\mu$ significantly reduces noise. Different levels of noise reduction can be achieved by changing the tuning parameter.

\section{DISCUSSION}

The iterative thresholding algorithm for linear inverse problems [1] was originally proposed for $\ell_{p}$-penalties with $1 \leq p \leq$ 2 and for any orthogonal decomposition. We have considered here the sparsity promoting $\ell_{1}$ penalty on a wavelet decomposition. In [56], it has been shown that the $\ell_{1}$ regularization performed significantly better than the $\ell_{2}$ regularization. The $\ell_{1}$ regularization could produce sparser representations while the $\ell_{2}$ regularization tends to blur edges.

The concensus among researchers is that the likelihood term has a great effect. However, in situations like ours where there is a strong regularization, the exact functional form of the data term is much less important. Yet, a topic of future research could be to develop a version of [1] for Poisson noise.

The modular structure of the iterative thresholding algorithm allows a flexible implementation. In particular, different wavelets can be used at a negligible implementation cost. The algorithm only needs to select the appropriate filters. In the case of the $E$-spline wavelets, these filters are level-dependent and need to be precalculated, introducing only a minor additional complexity to the implementation.

Interestingly the modular update scheme is similar to the expectation maximization smooth (EMS) or interiteration filtering
[57]-[59] reconstruction strategies, well known in the PET reconstruction community. The fundamental difference is the existence of strong convergence results for the iterative thresholding algorithm [1] while there is no proof of convergence for the EMS algorithm.

In our computer experiments, we introduced an artificial parameter $J_{s}$. In general one considers the same number of decomposition levels in all dimensions as we have done for the reconstruction of the clinical data. We have introduced this parameter solely to emphasize the effect of the $E$-spline wavelets in the temporal domain, e.g., $J_{s}=0$ corresponds to no spatial regularization. It is however natural to consider both temporal and spatial regularization and the balance between spatial and temporal smoothness can be adjusted by considering different scaling factors in the different subbands. For example, the subband-dependent threshold can be derived in a Bayesian framework when the wavelet coefficients in each subband are well modeled as realizations of a generalized Gaussian distribution [60].

We have compared the performance of the $E$-spline wavelets to the $B$-spline wavelets in our dynamic reconstruction task, for wavelets of the same order $N$. We expect that other classical wavelets would show results similar to the $B$-spline wavelets with equivalent order. In fact, the primary mathematical wavelet properties such as order of approximation and vanishing moments are entirely due to the $B$-spline component [30].

An important step that needs to be performed prior to the wavelet decomposition is the approximation of the reconstruction in the space $\mathcal{V}_{0}$. This step is implemented here using the 


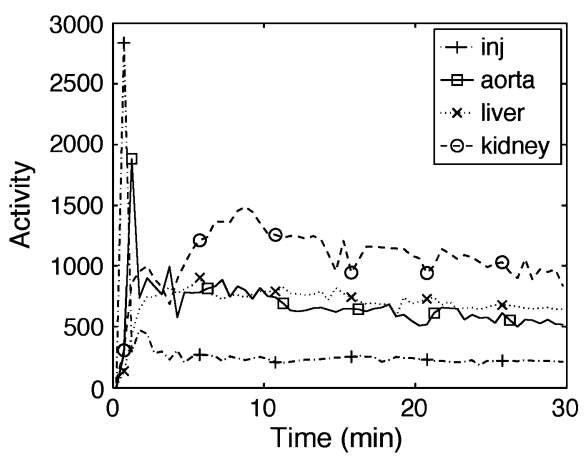

(a)

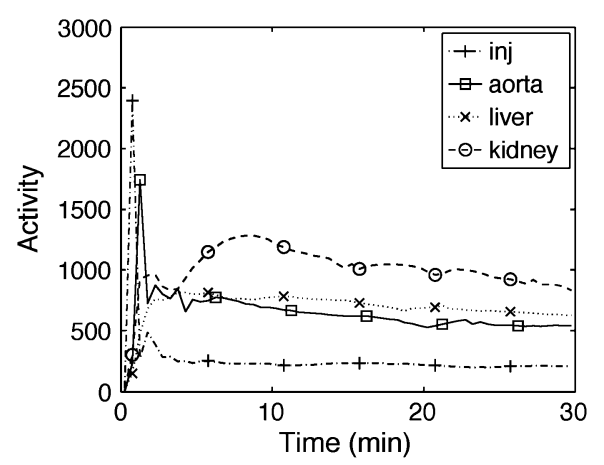

(b)

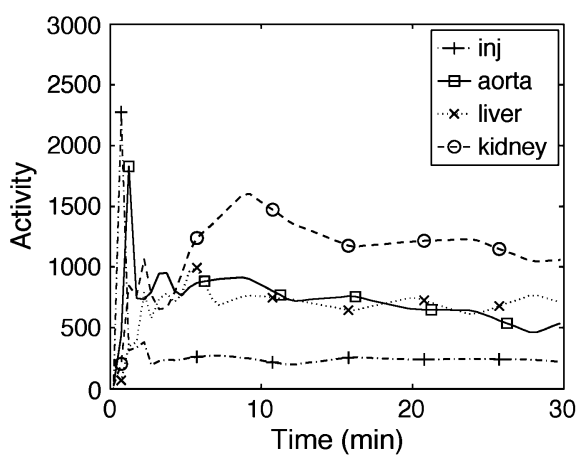

(c)

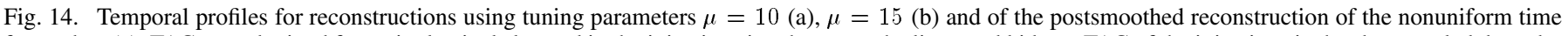

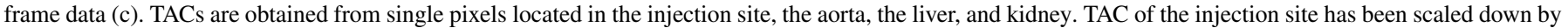
a factor 0.5 for a better display.

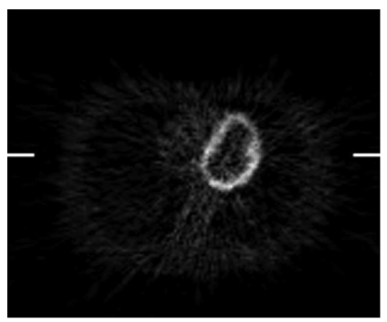

(a)

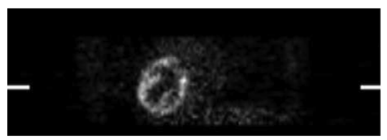

(c)

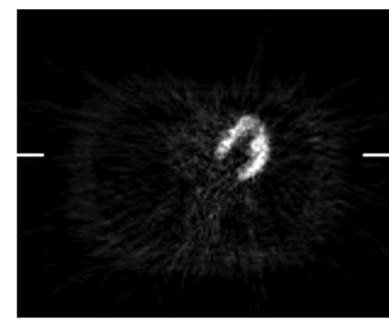

(b)

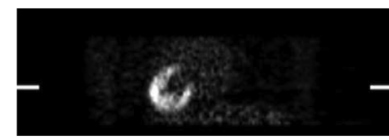

(d)
Fig. 15. Single slices for reconstructions using tuning parameter $\mu=0.1$. The slices are at $[(a),(c)] 0 \%$ and $[(b),(d)] 40 \%$ of the cardiac phase. Transaxial and transvers slices, [(a), (b)] and [(c), (d)], respectively. White bars illustrate the positions of the slices. Slices were cropped and the colorscale covers $0 \%-75 \%$ of the dynamic range of the total data set.

consistent sampling strategy which projects the pixelized reconstruction into $\mathcal{V}_{0}$. Another solution would be to discretize the problem directly in $\mathcal{V}_{0}$ by using $\phi_{0}^{(1)}(x)$ and $\phi_{0}^{(2)}(\tau)$ as basis functions instead of using time pixels. Similar discretizations were considered using modified Kaiser-Bessel basis functions [61], using $B$-splines in the spatial domain [62] and in the temporal domain [7], [9], [23]. If the data are available in list-mode format such a direct approach in the time domain is advantageous, as one can work directly with list-mode data without the need to bin the data in a number of frames. A disadvantage of the direct method is that in general the projection $\mathcal{N}$ onto the nonnegative functions is not implemented easily as it should be implemented directly in $\mathcal{V}_{0}$ rather than in $\mathcal{V}_{\zeta}$. A limitation related to using list-mode data would be that we have to perform a complete projection and backprojection to and from all sinogram bins for every event that is detected, as can be seen from (21). We have illustrated the selection of the $E$-spline wavelet defining parameters $\boldsymbol{\alpha}$ and $\boldsymbol{\gamma}$. We investigated the robustness of the parameter selection. Our results suggest that the method is not overly sensitive: there is a broad range of parameter vectors that perform better than $B$-spline wavelets. This was con-

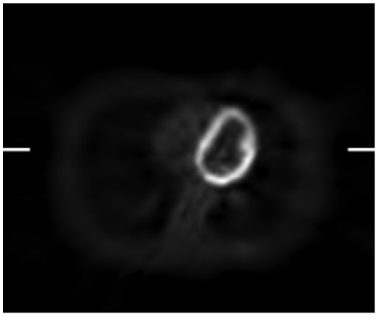

(a)

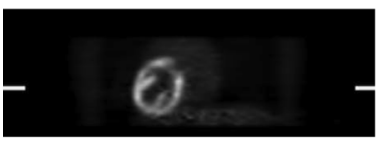

(c)

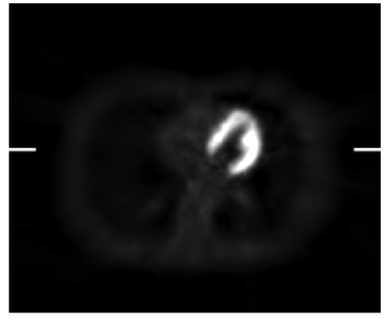

(b)

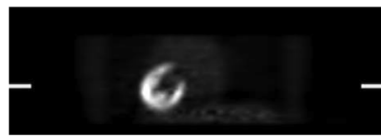

(d)
Fig. 16. Single slices for reconstructions using tuning parameter $\mu=0.3$. The slices are at $[(a),(c)] 0 \%$ and [(b), (d)] $40 \%$ of the cardiac phase. Transaxial and transvers slices, [(a), (b)] and [(c), (d)], respectively. White bars illustrate the positions of the slices. Slices were cropped and the colorscale covers $0 \%-75 \%$ of the dynamic range of the total data set.

firmed by both 1-D and tomographic simulations considering a wide range of imaging protocols (cardiac and brain imaging) and considering a range of parameter vectors. A fair amount of this robustness is necessary to allow the $E$-spline wavelets to be successfully applied in real-life situations. First, because the theoretically ideal parameter vectors are not know before reconstruction. Second, because different regions (blood/tissue, normal/disease) can have very different theoretically optimal parameters.

The presented link between the underlying operator $L_{\boldsymbol{\alpha}, \boldsymbol{\gamma}}$ of the $E$-spline wavelets and the differential equations modelling the TACs illustrates how the $E$-spline wavelets arise naturally in the setting of spatio-temporal PET reconstruction. Moreover it gives an insight in the process of selecting the parameter vectors. A careful selection is necessary to maximize the achieved gain compared to the $B$-spline wavelets. The presented parameter selection is still relatively crude and further investigation of this topic is appropriate. In particular, we have seen in our 1-D experiments that the optimal $\alpha$ in $\alpha$ was a little higher than the $\alpha$ derived from theoretical reasoning, especially in the low noise situations. 


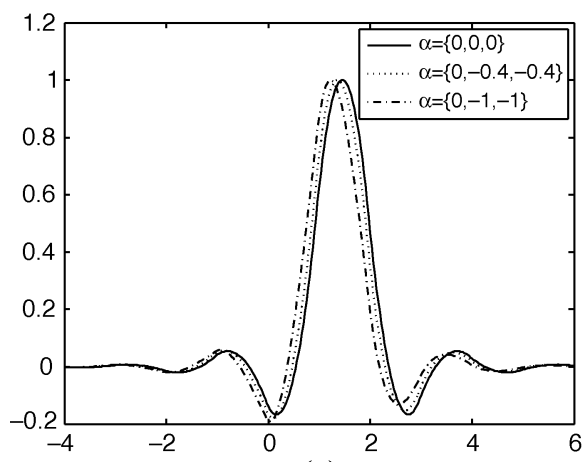

(a)

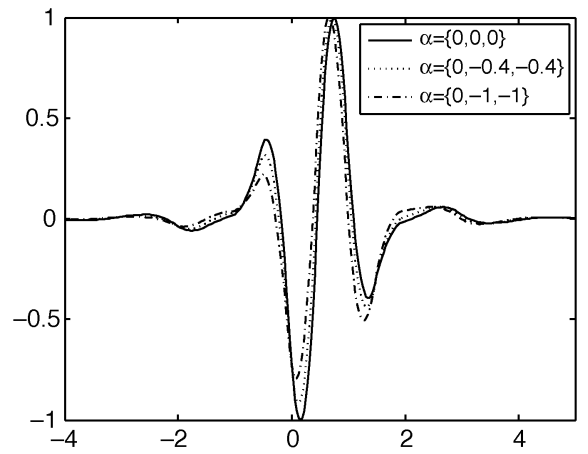

(c)

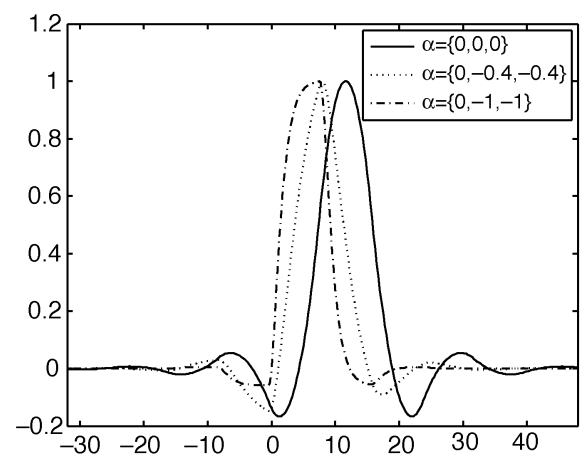

(b)

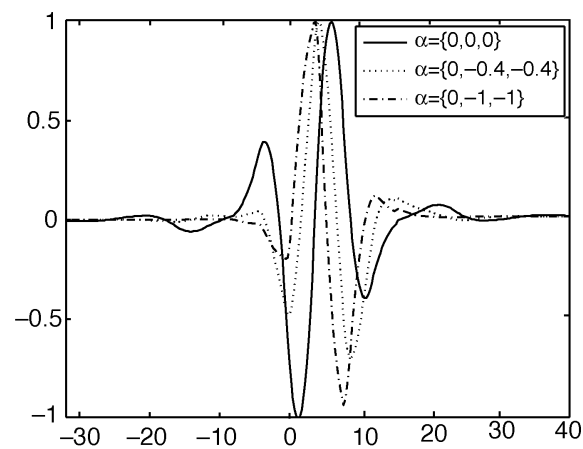

(d)

Fig. 17. $E$-spline wavelet examples at scales [(a), (c)] $j=0$ and [(b), (d) $] j=3$ for different parameter vectors $\boldsymbol{\alpha}$. [(a), (b)] Scaling functions and [(c), (d)] wavelets. For classical wavelets $(\boldsymbol{\alpha}=\{0,0,0\})$ the scaling functions and wavelets at different scales are obtained by dilating the wavelets and scaling functions at lower scales. This is not generally the case for $E$-spline wavelets $(\boldsymbol{\alpha}=\{0,-0.4,-0.4\}, \boldsymbol{\alpha}=\{0,-1,-1\})$.

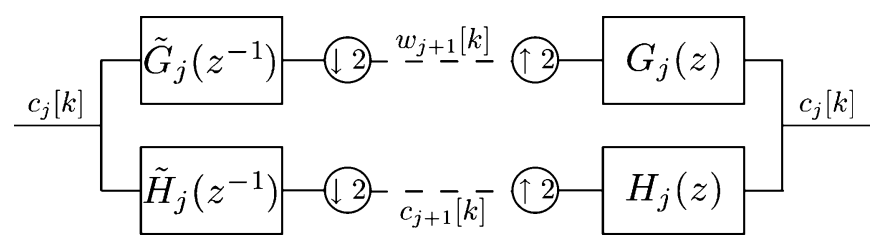

Fig. 18. Analysis and synthesis steps using one level of the filterbank. Scale-dependent filters (index $j$ ) are required for $E$-spline wavelets. For conventional wavelets, the filters are scale-independent, i.e., $\tilde{G}_{j}\left(z^{-1}\right)=$ $\tilde{G}\left(z^{-1}\right), \ldots, H_{j}(z)=H(z)$.

The results presented here are quite encouraging, but still preliminary, leaving still room for further research and improvements. Important topics include the selection of $E$-spline wavelet parameter vectors, selection of the threshold and appropriate modelling of the noise and wavelet coefficients, investigation of the convergence speed and possible speedup. Further studies are also required to examine the impact of the proposed method and to compare it to other techniques.

\section{CONCLUSION}

We have demonstrated the beneficial use of $E$-spline wavelets in combination with $\ell_{1}$ spatio-temporal regularization in dynamic PET imaging. The $E$-spline wavelets were found to be advantageous over conventional $B$-spline wavelets in modelling TACs.

The key concept is that the activity distribution in the body is ruled by systems of differential equations involving compartmental models. By construction, the proposed $E$-spline wavelets are well suited for the sparse representation of solutions of these differential equations. The parameters characterizing these wavelets are the poles and zeros of the underlying system. We have discussed the selection of the appropriate parameters and demonstrated that a wide range of these parameters outperformed the $B$-spline wavelets in terms of the reconstructed SNR and the sparsity of the wavelet coefficients. The experimental evaluation included 1-D denoising experiments and tomographic reconstruction experiments of simulated and clinical PET data.

The modular $\ell_{1}$ spatio-temporal regularization algorithm [1] allows a flexible selection of the wavelet basis. In combination with well designed $E$-spline wavelets this regularization algorithm is of interest for the quantitative nonparametric reconstruction of dynamic PET data.

\section{APPENDIX \\ E-SPLINE WAVELETS}

The $E$-spline wavelets $\psi_{j, k}^{(2)}(\tau)$ that we are using as the temporal basis functions are not wavelets in the classical sense [29], [63]. There are three main differences [21].

Operator-Like Behavior: While conventional wavelets act as pure derivatives, $E$-spline wavelets have a differential-operator-like behavior; i.e., when applied to the data the wavelet coefficients correspond to

$$
\left\langle\psi_{j, k}^{(2)}, f\right\rangle=\mathrm{L}_{\boldsymbol{\alpha}, \boldsymbol{\gamma}}\left\{f \star \phi_{j}^{(2)}\right\}\left(2^{j} k\right)
$$

where $\phi_{j}^{(2)}$ is the $E$-spline scaling function at level $j$ that acts as a low-pass smoothing function [21]. 
Dilation-Free Multi-Resolution: Conventional wavelets are obtained by dilation and translation of the wavelet $\psi^{(1)}(x)$, i.e., $\psi_{j, k}^{(1)}(x)=2^{-j / 2} \psi^{(1)}\left(x / 2^{j}-k\right)$. A similar expression holds for conventional scaling functions. For the $E$-spline wavelets, on the other hand, the wavelets and scaling functions at level $j+1$ are not dilations of the wavelets and scaling functions at level $j$. Some examples of $E$-spline wavelets and scaling functions at different levels $j$ are illustrated in Fig. 17.

Scale-Dependent Filters: The two-scale relation and wavelet equation [29], [30] are relaxed upon, allowing the filter coefficients $h_{j}[k]$ and $g_{j}[k]$ to depend on the scale $j$

$$
\begin{aligned}
\phi_{j+1}^{(2)}(\tau) & =\sum_{k} h_{j}[k] \phi_{j}^{(2)}\left(\tau-2^{j} k\right) \\
\psi_{j+1}^{(2)}(\tau) & =\sum_{k} g_{j}[k] \phi_{j}^{(2)}\left(\tau-2^{j} k\right) .
\end{aligned}
$$

Fortunately, the $E$-spline wavelet transform can still be efficiently calculated using a two-channel filterbank after approximating the signal in $\mathcal{V}_{0}$ [21]. However, the low $\left(h_{j}[k]\right)$ and high $\left(g_{j}[k]\right)$ pass filters will depend on the scale, as illustrated in Fig. 18. This translates into selecting the appropriate precalculated filters at every decomposition iteration. Explicit expressions for designing the $E$-spline wavelet filters can be found in [21]. For orthonormalized $E$-spline wavelets the filters are IIR with exponential decay.

The gain we get from this extension is that we can generalize the property of the vanishing moments (see Section III-A).

\section{ACKNOWLEDGMENT}

The authors would like to thank Dr. P. Millet of the Geneva University Hospital who provided us with the input curves for the FMZ data, Dr. P. Croisille of the Geneva University Hospital who provided the gated PET data, Dr. H. Zaidi of the Geneva University Hospital who has helped us with the Siemens data format and the calculation of the attenuation map, and Dr. I. Goethals of the Ghent University Hospital who provided the dynamic liver PET data.

\section{REFERENCES}

[1] I. Daubechies, M. Defrise, and C. De Mol, "An iterative thresholding algorithm for linear inverse problems with a sparsity constraint," Commun. Pure Appl. Math., vol. 57, no. 11, pp. 1413-1457, 2004.

[2] R. M. Lewitt and S. Matej, "Overview of methods for image reconstruction from projections in emission computed tomography," Proc. IEEE, vol. 91, no. 10, pp. 1588-1611, Oct. 2003.

[3] J. Y. Qi and R. M. Leahy, "Iterative reconstruction techniques in emission computed tomography," Phys. Med. Biol., vol. 51, no. 15, pp. R541-R578, Aug. 2006.

[4] T. Hebert and R. Leahy, "A generalized EM algorithm for 3-D Bayesian reconstruction from poisson data using Gibbs priors," IEEE Trans. Med. Imag., vol. 8, no. 2, pp. 194-202, Jun. 1989.

[5] J. A. Fessler, "Penalized weighted least-squares image-reconstruction for positron emission tomography," IEEE Trans. Med. Imag., vol. 13, no. 2, pp. 290-300, Jun. 1994.

[6] J. A. Fessler, "Hybrid poisson/polynomial objective functions for tomographic image reconstruction from transmission scans," IEEE Trans. Image Process., vol. 4, no. 10, pp. 1439-1450, Oct. 1995.

[7] T. E. Nichols, J. Qi, E. Asma, and R. M. Leahy, "Spatiotemporal reconstruction of list-mode PET data," IEEE Trans. Med. Imag., vol. 21, no. 4, pp. 396-404, Apr. 2002.

[8] Q. Li, E. Asma, S. Ahn, and R. M. Leahy, "A fast fully 4-D incremental gradient reconstruction algorithm for list mode PET data," IEEE Trans. Med. Imag., vol. 26, no. 1, pp. 58-67, Jan. 2007.
[9] J. Verhaeghe, Y. D'Asseler, S. Vandenberghe, S. Staelens, and I. Lemahieu, "An investigation of temporal regularization techniques for dynamic PET reconstructions using temporal splines," Med. Phys., vol. 34, no. 5, pp. 1525-1875, May 2007.

[10] N. M. Alpert, A. Reilhac, T. C. Chio, and I. Selesnick, "Optimization of dynamic measurement of receptor kinetics by wavelet denoising," NeuroImage, vol. 30, no. 2, pp. 444-451, Apr. 2006.

[11] P. Millet, V. Ibanez, J. Delforge, S. Pappata, and J. Guimon, "Wavelet analysis of dynamic PET data: Application to the parametric imaging of benzodiazepine receptor concentration," Neurolmage, vol. 11, no. 5, pp. 458-472, May 2000.

[12] F. E. Turkheimer, M. Brett, D. Visvikis, and V. J. Cunningham, "Multiresolution analysis of emission tomography images in the wavelet domain," J. Cereb. Blood Flow Metab., vol. 19, no. 11, pp. 1189-1208, Nov. 1999.

[13] D. L. Donoho, "Nonlinear solution of linear inverse problems by wavelet-vaguelette decomposition," Appl. Computational Harmonic Anal., vol. 2, no. 2, pp. 101-126, Apr. 1995.

[14] E. D. Kolaczyk, "A wavelet shrinkage approach to tomographic image reconstruction," J. Am. Stat. Assoc., vol. 91, no. 435, pp. 1079-1090, Sep. 1996.

[15] F. Abramovich and B. W. Silverman, "Wavelet decomposition approaches to statistical inverse problems," Biometrika, vol. 85, no. 1 , pp. 115-129, Mar. 1998.

[16] D. L. Donoho, "De-noising by soft-thresholding," IEEE Trans. Inf. Theory, vol. 41, no. 3, pp. 613-627, May 1995.

[17] J. L. Starck, M. K. Nguyen, and F. Murtagh, "Wavelets and curvelets for image deconvolution: A combined approach," Signal Process., vol. 83, no. 10, pp. 2279-2283, Oct. 2003.

[18] M. A. T. Figueiredo and R. D. Nowak, "An EM algorithm for waveletbased image restoration," IEEE Trans. Image Process., vol. 12, no. 8, pp. 906-916, Aug. 2003.

[19] T. Frese, C. A. Bouman, and K. Sauer, "Adaptive wavelet graph model for bayesian tomographic reconstruction," IEEE Trans. Image Process., vol. 11, no. 7, pp. 756-770, Jul. 2002.

[20] R. D. Nowak and E. D. Kolaczyk, "A statistical multiscale framework for poisson inverse problems," IEEE Trans. Inf. Theory, vol. 46, no. 5, pp. 1811-1825, Aug. 2000.

[21] I. Khalidov and M. Unser, "From differential equations to the construction of new wavelet-like bases," IEEE Trans. Signal Process., vol. 54, no. 4, pp. 1256-1267, Apr. 2006.

[22] D. L. Snyder, "Parameter estimation for dynamic studies in emission tomography systems having list-mode data," IEEE Trans. Nucl. Sci., vol. NS-31, no. 2, pp. 925-931, 1984.

[23] B. W. Reutter, G. T. Gullberg, and R. H. Huesman, "Direct leastsquares estimation of spatiotemporal distributions from dynamic SPECT projections using a spatial segmentation and temporal B-splines," IEEE Trans. Med. Imag., vol. 19, no. 5, pp. 434-450, May 2000.

[24] A. J. Reader, F. C. Sureau, C. Comtat, R. Trebossen, and I. Buvat, "Joint estimation of dynamic PET images and temporal basis functions using fully 4D ML-EM," Phys. Med. Biol., vol. 51, no. 21, pp. 5455-5474, Nov. 2006

[25] J. Matthews, D. Bailey, P. Price, and V. Cunningham, "The direct calculation of parametric images from dynamic PET data using maximumlikelihood iterative reconstruction," Phys. Med. Biol., vol. 42, no. 6, pp. 1155-1173, 1997.

[26] Y. S. Xu, J. B. Weaver, D. M. Healy, and J. Lu, "Wavelet transform domain filters-A spatially selective noise filtration technique," IEEE Trans. Image Process., vol. 3, no. 6, pp. 747-758, Nov. 1994.

[27] M. Unser and A. Aldroubi, "A review of wavelets in biomedical applications," Proc. IEEE, vol. 84, no. 4, pp. 626-638, Apr. 1996.

[28] D. L. Donoho and X. M. Huo, "Uncertainty principles and ideal atomic decomposition," IEEE Trans. Inf. Theory, vol. 47, no. 7, pp. 2845-2862, Nov. 2001.

[29] S. G. Mallat, "A theory for multiresolution signal decomposition: The wavelet representation," IEEE Trans. Pattern Anal. Mach. Intell., vol. 11, no. 7, pp. 674-693, Jul. 1989.

[30] M. Unser and T. Blu, "Wavelet theory demystified," IEEE Trans. Signal Process., vol. 51, no. 2, pp. 470-483, Feb. 2003.

[31] M. Unser and T. Blu, "Cardinal exponential splines: Part I-Theory and filtering algorithms," IEEE Trans. Signal Process., vol. 53, no. 4, pp. 1425-1438, Apr. 2005.

[32] M. Unser, "Cardinal exponential splines: Part II-Think analog, act digital," IEEE Trans. Signal Process., vol. 53, no. 4, pp. 1439-1449, Apr. 2005.

[33] S. R. Cherry, J. A. Sorensen, and M. E. Phelps, Physics in Nuclear Medicine, 3rd ed. Philadelphia, PA: Saunders, 2003, ch. 20, pp. 377-403. 
[34] R. N. Gunn, S. R. Gunn, and V. J. Cunningham, "Positron emission tomography compartmental models," J. Cereb. Blood Flow Metab., vol. 21, no. 6, pp. 635-652, Jun. 2001.

[35] M. Slifstein and M. Laruelle, "Models and methods for derivation of in vivo neuroreceptor parameters with PET and SPECT reversible radiotracers," Nucl. Med. Biol., vol. 28, no. 5, pp. 595-608, Jul. 2001.

[36] M. Bentourkia and H. Zaidi, Quantitative Analysis in Nuclear Medicine Imaging. New York: Springer, 2006, ch. 12, pp. 391-413.

[37] L. Sokoloff, M. Reivich, C. Kennedy, M. H. Desrosiers, C. S. Patlak, K. D. Pettigrew, O. Sakurada, and M. Shinohara, "The $\left[{ }^{14} \mathrm{C}\right]$ deoxyglucose method for measurement of local cerebral glucoseutilization-Theory, procedure, and normal values in conscious and anesthetized albinorat," J. Neurochem., vol. 28, no. 5, pp. 897-916, 1977.

[38] M. E. Phelps, S. C. Huang, E. J. Hoffman, C. Selin, L. Sokoloff, and D. E. Kuhl, "Tomographic measurement of local cerebral glucose metabolic-rate in humans with (F-18)2-Fluoro-2-Deoxy-D-Glucose-validation of method," Ann. Neurol., vol. 6, no. 5, pp. 371-388, 1979.

[39] M. C. Asselin, L. M. Wahl, V. J. Cunningham, S. Amano, and C. Nahmias, "In vivo metabolism and partitioning of 6-[F-18]fluoro-1-metatyrosine in whole blood: A unified compartment model," Phys. Med. Biol., vol. 47, no. 11, pp. 1961-1977, Jun. 2002.

[40] R. Davenport, "The derivation of the gamma-variate relationship for tracer dilution curves," J. Nucl. Med., vol. 24, no. 10, pp. 945-948, 1983.

[41] D. Feng, S. C. Huang, and X. M. Wang, "Models for computersimulation studies of input functions for tracer kinetic modeling with positron emission tomography," Int. J. Bio-Med. Comput., vol. 32, no. 2, pp. 95-110, Mar. 1993

[42] M. M. Graham, "Physiologic smoothing of blood time-activity curves for PET data analysis," J. Nucl. Med., vol. 38, no. 7, pp. 1161-1168, Jul. 1997.

[43] S. R. Golish, J. D. Hove, H. R. Schelbert, and S. S. Gambhir, "A fast nonlinear method for parametric imaging of myocardial perfusion by dynamic N-13-ammonia PET," J. Nucl. Med., vol. 42, no. 6, pp. 924-931, 2001.

[44] J. A. Thompson, F. Starmar, R. E. Whalen, and H. D. McIntosh, "Indicator transit time considered as a gamma variate," Circ. Res., vol. 14, pp. 502-515, 1964.

[45] D. L. Donoho and I. M. Johnstone, "Adapting to unknown smoothness via wavelet shrinkage," J. Am. Stat. Assoc., vol. 90, no. 432, pp. 1200-1224, Dec. 1995.

[46] A. Chambolle, R. A. DeVore, N. Y. Lee, and B. J. Lucier, "Nonlinear wavelet image processing: Variational problems, compression, and noise removal through wavelet shrinkage," IEEE Trans. Image Process., vol. 7, no. 3, pp. 319-335, Mar. 1998.

[47] M. Unser and A. Aldroubi, "A general sampling theory for nonideal acquisition devices," IEEE Trans. Signal Process., vol. 42, no. 11, pp. 2915-2925, Nov. 1994.

[48] P. Millet, C. Graf, A. Buck, B. Walder, and V. Ibanez, "Evaluation of the reference tissue models for PET and SPECT benzodiazepine binding parameters," NeuroImage, vol. 17, no. 2, pp. 928-942, Oct. 2002.
[49] W. P. Segars, D. S. Lalush, and B. M. W. Tsui, "A realistic spline-based dynamic heart phantom," IEEE Trans. Nucl. Sci., vol. 46, no. 3, pp. 503-506, Jun. 1999.

[50] I. G. Zubal, C. R. Harrell, E. O. Smith, Z. Rattner, G. Gindi, and P. B. Hoffer, "Computerized 3-dimensional segmented human anatomy," Med. Phys., vol. 21, no. 2, pp. 299-302, Feb. 1994.

[51] J. Delforge, S. Pappata, P. Millet, Y. Samson, B. Bendriem, A. Jobert, C. Crouzel, and A. Syrota, "Quantification of benzodiazepine receptors in human brain using PET, [C-11] flumazenil, and a single-experiment protocol," J. Cereb. Blood Flow Metab., vol. 15, no. 2, pp. 284-300, Mar. 1995.

[52] S. Surti and J. S. Karp, "Imaging characteristics of a 3-dimensional GSO whole-body PET camera," J. Nucl. Med., vol. 45, no. 6, pp. 1040-1049, 2004.

[53] M. E. Daube-Witherspoon and G. Muehllehner, "Treatment of axial data in 3-dimensional PET," J. Nucl. Med., vol. 28, no. 11, pp. 1717-1724, Nov. 1987.

[54] M. Brambilla, C. Secco, M. Dominietto, R. Matheoud, G. Sacchetti, and E. Inglese, "Performance characteristics obtained for a new 3-dimensional lutetium oxylorthosilicate-based whole-body PET/CT scanner with the national electrical manufacturers association NU 2-2001 standard," J. Nucl. Med., vol. 46, no. 12, pp. 2083-2091, Dec. 2005.

[55] J. P. J. Carney, D. W. Townsend, V. Rappoport, and B. Bendriem, "Method for transforming ct images for attenuation correction in pet/ct imaging," Med. Phys., vol. 33, no. 4, pp. 976-983, Apr. 2006.

[56] I. Loris, G. Nolet, I. Daubechies, and F. Dahlen, "Tomographic inversion using 11-norm regularization of wavelet coefficients," Geophys. J. Int., vol. 170, no. 1, pp. 359-370, Jul. 2007.

[57] B. W. Silverman, M. C. Jones, J. D. Wilson, and D. W. Nychka, "A smoothed EM approach to indirect estimation problems, with particular reference to stereology and emission tomography," J. R. Stat. Soc. Series B-Methodological, vol. 52, no. 2, pp. 271-324, 1990.

[58] J. Llacer, E. Veklerov, K. J. Coakley, E. J. Hoffman, and J. Nunez, "Statistical-analysis of maximum-likelihood estimator images of human brain FDG PET studies," IEEE Trans. Med. Imag., vol. 12, no. 2, pp. 215-231, Jun. 1993.

[59] E. T. P. Slijpen and F. J. Beekman, "Comparison of post-filtering and filtering between iterations for SPECT reconstruction," IEEE Trans. Nucl. Sci., vol. 46, no. 6, pp. 2233-2238, Dec. 1999.

[60] S. G. Chang, B. Yu, and M. Vetterli, "Adaptive wavelet thresholding for image denoising and compression," IEEE Trans. Image Process., vol. 9, no. 9, pp. 1532-1546, Sep. 2000.

[61] R. M. Lewitt, "Alternatives to voxels for image representation in iterative reconstruction algorithms," Phys. Med. Biol., vol. 37, no. 3, pp. 705-716, 1992

[62] S. Horbelt, M. Liebling, and M. Unser, "Discretization of the radon transform and of its inverse by spline convolutions," IEEE Trans. Med. Imag., vol. 21, no. 4, pp. 363-376, Apr. 2002.

[63] S. Mallat, A Wavelet Tour of Signal Processing. San Diego, CA: Academic, 1998. 